\title{
Channelized free-surface flow of cohesionless granular avalanches in a chute with shallow lateral curvature
}

\author{
By M. WIELAND ${ }^{1}$, J. M. N. T. GRAY ${ }^{1,2}$ AND K. HUTTER \\ ${ }^{1}$ Institut für Mechanik, Technische Universität Darmstadt, 64289 Darmstadt, Germany \\ ${ }^{2}$ Department of Mathematics, University of Manchester, Manchester M13 9PL, UK
}

(Received 29 November 1997 and in revised form 15 February 1999)

A series of laboratory experiments and numerical simulations have been performed to investigate the rapid fluid-like flow of a finite mass of granular material down a chute with partial lateral confinement. The chute consists of a section inclined at $40^{\circ}$ to the horizontal, which is connected to a plane run-out zone by a smooth transition. The flow is confined on the inclined section by a shallow parabolic cross-slope profile. Photogrammetric techniques have been used to determine the position of the evolving boundary during the flow, and the free-surface height of the stationary granular deposit in the run-out zone. The results of three experiments with different granular materials are presented and shown to be in very good agreement with numerical simulations based on the Savage-Hutter theory for granular avalanches. The basal topography over which the avalanche flows has a strong channelizing effect on the inclined section of the chute. As the avalanche reaches the run-out zone, where the lateral confinement ceases, the head spreads out to give the avalanche a characteristic 'tadpole' shape. Sharp gradients in the avalanche thickness and velocity began to develop at the interface between the nose and tail of the avalanche as it came to rest, indicating that a shock wave develops close to the end of the experiments.

\section{Introduction}

Snow slab avalanches, landslides and rock falls are extremely dangerous and destructive natural phenomena, whose frequency and amplitude appears to have increased during the past two decades. A reliable method of predicting avalanche paths and maximum run-out distances is therefore of considerable interest to civil engineers responsible for planning and development in populated mountainous regions. The Savage-Hutter $(1989,1991)$ theory for the gravity-driven, free-surface flow of a finite mass of granular material over a rough inclined base has been established as the leading model for this purpose.

The theory was originally formulated (Savage \& Hutter 1989) for an incompressible granular avalanche flowing down an inclined plane. The balance equations were formulated for two-dimensional plane deformations, and subsequently reduced by the shallowness approximation to obtain a one-dimensional theory by integrating through the avalanche depth. The effects of basal friction were parameterized by a Coulomb-type rate-independent dry friction law with basal angle of friction, $\delta$, and the downslope avalanche velocity was assumed to be independent of depth. This theory was found to be in excellent agreement with laboratory experiments (Savage 


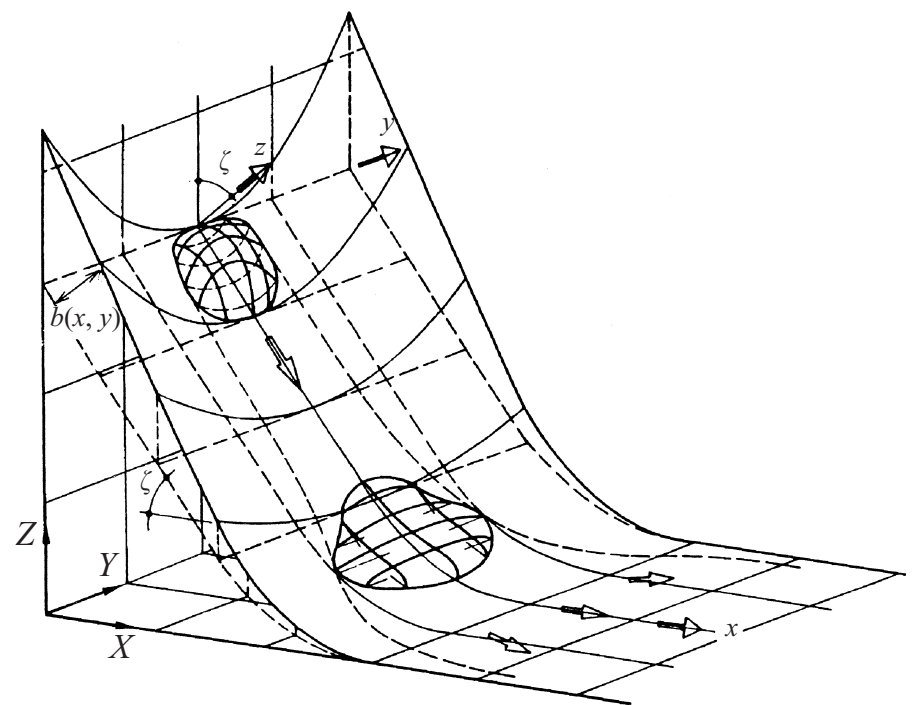

FIGURE 1. The simple curvilinear coordinate system $O x y z$ consists of an inclined plane connected to a horizontal plane by a smooth transition. The local inclination angle of the curvilinear surface $z=0$ (dashed lines) is $\zeta$. The actual basal topography $z=b(x, y)$ (solid lines) is superposed on the $z=0$ curvilinear surface.

\& Hutter 1989; Hutter \& Koch 1991; Greve \& Hutter 1993). A two-dimensional generalization of the theory for planar topographies (Hutter et al. 1993; Greve, Koch $\&$ Hutter 1994) has also yielded very good results (Koch, Greve \& Hutter 1994).

The Savage-Hutter theory has recently been extended (Gray, Wieland \& Hutter 1999) to model the flow of granular avalanches over a wide range of shallow threedimensional topographies. The resulting depth-integrated (two-dimensional) theory allows the motion of granular avalanches to be modelled from initiation to run-out on most naturally occurring slopes and hence provides a method of predicting terrain specific information for avalanche risk assessment. To test this theory three laboratory experiments are performed on a chute with partial lateral confinement, and the results are compared to numerical simulations.

\section{Governing equations}

The two-dimensional depth-integrated equations used in this paper were derived by Gray et al. (1999). A reference surface that follows the mean downslope bed topography is used to define a plane orthogonal curvilinear coordinate system, Oxyz. The $z$-axis is normal to the reference surface and the $x$ - and $y$ coordinates are tangential to it, with the $x$-axis oriented downslope. The downslope inclination angle $\zeta$ is used to define the reference surface as a function of the downslope coordinate $x$. The reference surface does not vary as a function of the cross-slope coordinate $y$. The chute geometry is superposed by defining its height $z=b(x, y)$ above the reference surface, $z=0$, as illustrated in figure 1 . For notational simplicity components in the $x$-direction are referred to as downslope and components in the $y$-direction as cross-slope, even though the local downslope direction of the basal topography may not coincide with the direction of the $x$-coordinate.

The depth-integrated mass balance equation for an incompressible material together with the kinematic boundary conditions at the free surface and base of the avalanche 
yield a continuity equation for the avalanche thickness $h$

$$
\frac{\mathrm{d} h}{\mathrm{~d} t}+h\left(\frac{\partial u}{\partial x}+\frac{\partial v}{\partial y}\right)=0
$$

where $\boldsymbol{v}=(u, v)$ is the depth-averaged surface-parallel velocity with components $u, v$ in the down- and cross-slope directions, respectively. The two-dimensional advective derivative $\mathrm{d} / \mathrm{d} t=\partial / \partial t+u \partial / \partial x+v \partial / \partial y$. The momentum balance equations reduce to

$$
\begin{gathered}
\frac{\mathrm{d} u}{\mathrm{~d} t}=\sin \zeta-\frac{u}{|\boldsymbol{v}|} \tan \delta\left(\cos \zeta+\lambda \kappa u^{2}\right)-\epsilon \cos \zeta\left(K_{x} \frac{\partial h}{\partial x}+\frac{\partial b}{\partial x}\right), \\
\frac{\mathrm{d} v}{\mathrm{~d} t}=-\frac{v}{|\boldsymbol{v}|} \tan \delta\left(\cos \zeta+\lambda \kappa u^{2}\right)-\epsilon \cos \zeta\left(K_{y} \frac{\partial h}{\partial y}+\frac{\partial b}{\partial y}\right),
\end{gathered}
$$

where $\delta$ is the bed friction angle and $\kappa=-\partial \zeta / \partial x$ is the local curvature of the reference surface. The Earth pressure coefficients $K_{x}$ and $K_{y}$ are equal to the ratio of the in-plane to vertical pressure in the down- and cross-slope directions, respectively. That is, $K_{x}=p_{x x} / p_{z z}$ and $K_{y}=p_{y y} / p_{z z}$. The conservation equations (2.1)-(2.3) are presented in non-dimensional form. The non-dimensional variables, $h, b, x, y, u, v$ and $\kappa$, can be mapped back to their physical counterparts, $\tilde{h}, \tilde{b}, \tilde{x}, \tilde{y}, \tilde{u}, \tilde{v}$ and $\tilde{\kappa}$, by applying the scalings

$$
(\tilde{x}, \tilde{y})=L(x, y), \quad(\tilde{h}, \tilde{b})=H(h, b), \quad \tilde{t}=\sqrt{L / g} t, \quad \tilde{\kappa}=\kappa / \mathscr{R}, \quad(\tilde{u}, \tilde{v})=\sqrt{g L}(u, v),
$$

where $g$ is the constant of gravitational acceleration, $H$ is a typical avalanche thickness, $L$ is a typical length and $\mathscr{R}$ is a typical radius of curvature of the chute in the downslope direction. The Savage-Hutter theory assumes that both the aspect ratio of the avalanche $\epsilon=H / L$ and the characteristic curvature of the chute $\lambda=L / \mathscr{R}$, arising in equation (2.2) and (2.3), are small.

A key feature of the Savage-Hutter (1989) theory was the derivation of an expression for the downslope Earth pressure coefficient $K_{x}$, based on a Mohr-Coulomb constitutive relation for the granular nature of the material. Hutter et al. (1993) generalized this for the two-dimensional depth-integrated case to yield limiting values for both $K_{x}$ and $K_{y}$ in dilatation and compression. These relations are valid only when the motion is chiefly downhill and the shearing in the $(x, y)$-plane is small in comparison with the shearing in the $(x, z)$ - and $(y, z)$-planes. When the sidewise motion is large or when there is strong lateral confinement between rough walls these assumptions, of course, break down. However, in the experiments and simulations performed in this paper the chute has shallow lateral curvature, so the sidewise confinement is small, and the predominant motion is downslope. The Earth pressure coefficient relations derived by Hutter et al. (1993) are therefore assumed here.

The coefficients $K_{x}$ and $K_{y}$ are piecewise constant and are described as being active, or passive, dependent on whether the motion is dilatational, or compressional

$$
K_{x}= \begin{cases}K_{x_{a c t}}, & \partial u / \partial x \geqslant 0 \\ K_{x_{p a s}}, & \partial u / \partial x<0\end{cases}
$$

and

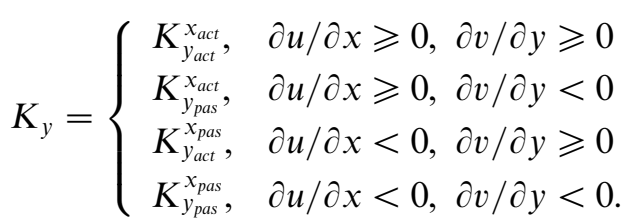


Their values are given by

$$
\begin{aligned}
& K_{x_{\text {act } \text { pas }}}=2 \sec ^{2} \phi\left(1 \mp \sqrt{1-\cos ^{2} \phi \sec ^{2} \delta}\right)-1, \\
& K_{y_{\text {act } / \text { pas }}}^{x}=\frac{1}{2}\left(K_{x}+1 \mp \sqrt{\left(K_{x}-1\right)^{2}+4 \tan ^{2} \delta}\right),
\end{aligned}
$$

where $\phi$ is the internal angle of friction of the granular material. The approximation that the downslope motion dominates over most of the avalanche track destroys the rotational invariance of the Earth pressure coefficients, but yields a relatively simple system of equations that is favoured at this stage. The magnitude of these terms plays an important role in the development of the avalanche shape, as they control how much spreading and contraction occur.

\section{Experimental setup}

The laboratory experiments were performed on a specially constructed chute. The reference surface consists of a plane inclined at $40^{\circ}$ to the horizontal, which is connected to a horizontal run-out zone by a curved transition region. The threedimensional basal topography over which the avalanche flows is then superposed normal to the reference surface. Prior to release the granular material fills a Perspex cap that sits flush with the parabolic cross-slope topography. The avalanche is initiated by rapidly raising the cap and it is then accelerated by the component of gravity acting in the downslope direction. When the avalanche enters the run-out zone its energy is rapidly dissipated by the basal friction and it quickly comes to rest. Typical run times are of the order of 1-2s with travel distances of up to $4 \mathrm{~m}$. A series of photographs from a typical experiment is shown in figure 2 .

\subsection{Slope-fitted curvilinear coordinates}

The reference surface is defined by the variation of its inclination angle, $\zeta$, with the downslope coordinate $x$. A relatively simple set of reference coordinates is used in the simulations, which reflects the mean downslope inclination angle of the experimental chute

$$
\zeta= \begin{cases}\zeta_{0}, & x<x_{a} \\ \zeta_{0}\left(x_{b}-x\right) /\left(x_{b}-x_{a}\right), & x_{a} \leqslant x \leqslant x_{b} \\ 0, & x_{b}<x,\end{cases}
$$

where $\zeta_{0}=40^{\circ}$. The origin is placed close to the release point and the beginning of the transition zone (and end of the parabolic chute) lies at $\tilde{x}_{a}=175 \mathrm{~cm}$, whilst the end of the transition region (and beginning of the horizontal flat plane) lies at $\tilde{x}_{b}=215 \mathrm{~cm}$. The mapping from the curvilinear system back to rectangular coordinates is given in Appendix A.

\subsection{Superposed basal topography}

A shallow parabolic cross-slope profile, with radius of curvature $\tilde{R}=110 \mathrm{~cm}$ is prescribed on the inclined section of the the chute, $x<x_{a}$. This opens out into a flat run-out zone in the region, $x>x_{b}$, and in the transition zone, $x_{a} \leqslant x \leqslant x_{b}$, a continuous differentiable function is constructed to provide a smooth change in the topography. The parabolic section and the flat run-out plane are both constructed from sheet steel and the transition is built from wood and fine modelling plaster. The entire chute was then sprayed with paint to give it a smooth finish with an even bed friction angle. 

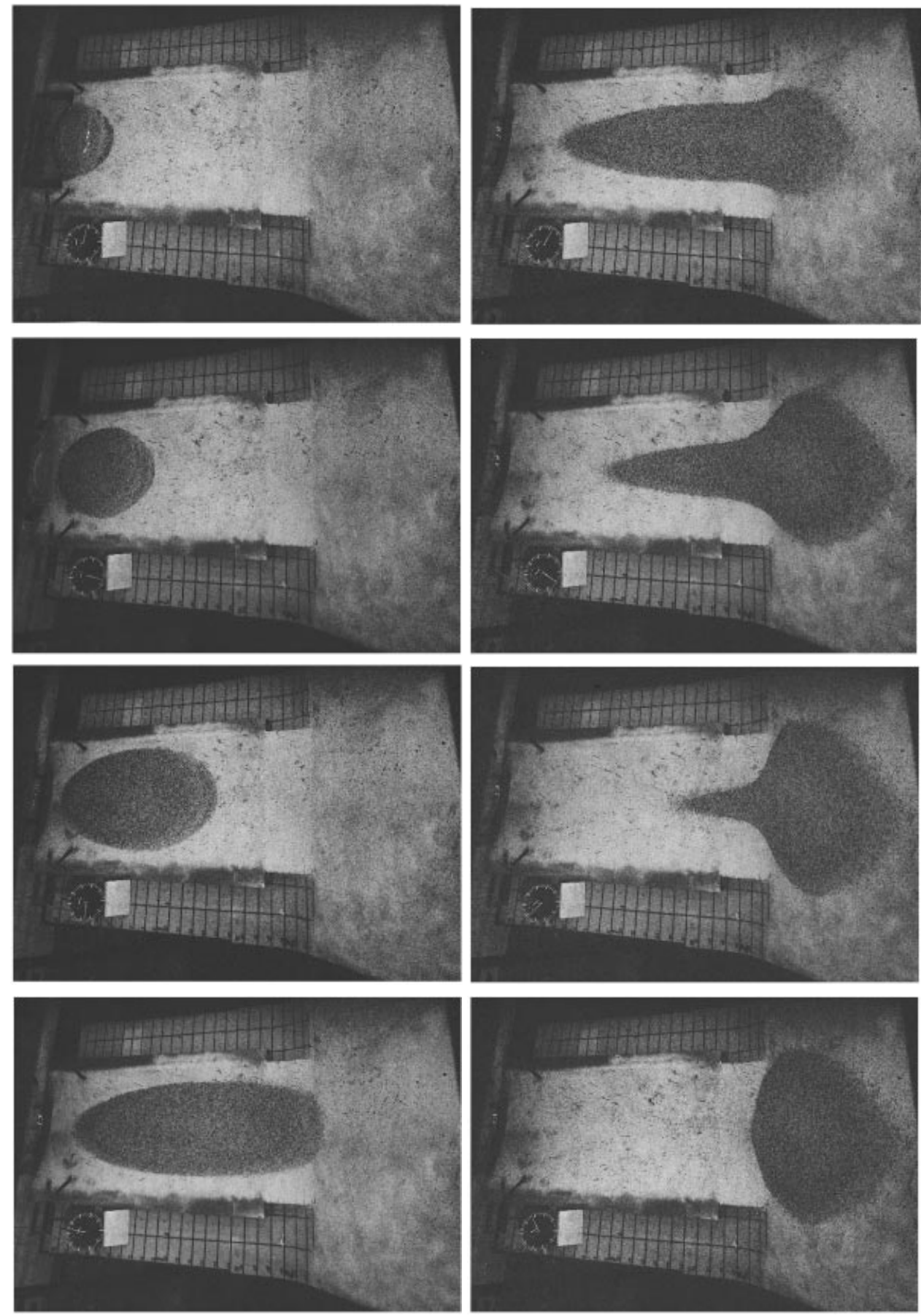

FIGURE 2. An experimental image sequence from experiment V05 showing the deformation of the avalanche at approximately $1 / 4 \mathrm{~s}$ time intervals. The granular material flows downhill from left to right. The parabolic chute lies is on the left-hand and the horizontal run-out plane is on the right-hand side of each picture. The image sequence begins in the top left-hand panel and ends in the bottom right, going from top to bottom, and the large hand of the clock performs one revolution every second. 


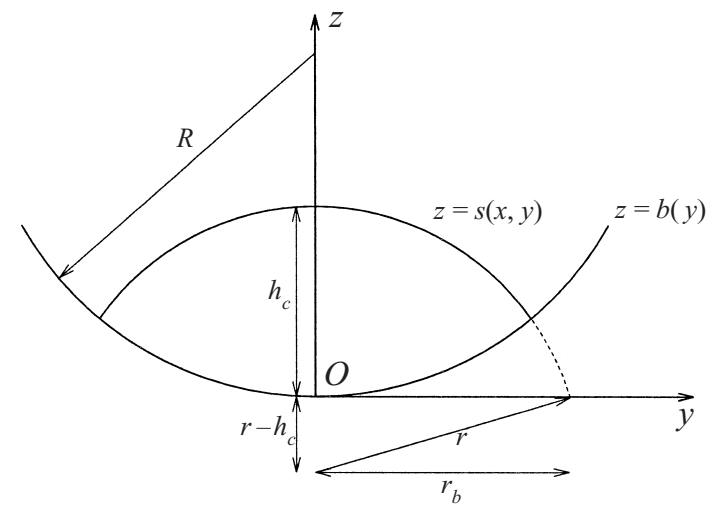

FIGURE 3. A cross-slope section through the centre of the spherical cap that is used to generate the initial free surface of the granular material. The chute has a shallow parabolic cross-slope profile with radius of curvature $R$, whilst the spherical cap has radius $r$ and maximum height $h_{c}$ above the basal topography. If $R \rightarrow \infty$ the edge of the avalanche domain is circular with radius $r_{b}$.

The functional form of the chute topography, $b$, is

$$
b= \begin{cases}\frac{y^{2}}{2 R}, & x<x_{a} \\ \frac{y^{2}}{2 R}\left\{3\left(\frac{x-x_{b}}{x_{a}-x_{b}}\right)^{2}-2\left(\frac{x-x_{b}}{x_{a}-x_{b}}\right)^{3}\right\}, & x_{a} \leqslant x \leqslant x_{b} \\ 0, & x_{b}<x .\end{cases}
$$

In the channel section $\left(x<x_{a}\right)$ of the chute the basal topography is a function of $y$ only, whilst in the transition zone $\left(x_{a} \leqslant x \leqslant x_{b}\right)$ the basal topography is a function of both $x$ and $y$. In the run-out zone $\left(x>x_{b}\right)$ the basal topography is independent of both $x$ and $y$.

\subsection{Initial conditions}

The granular material is released from a hemi-spherical Perspex cap at the top of the chute, which is fitted to the parabolic cross-slope basal topography. The initial free surface, $s$, of the granular material is

$$
s(x, y, 0)=\left(r^{2}-x^{2}-y^{2}\right)^{1 / 2}-\left(r-h_{c}\right),
$$

where $r$ is the radius of the sphere and $h_{c}$ is the maximum height of the free surface above the chute. The basal topography in the channel section of the chute is given by (3.2), and the avalanche boundary lies at the intersection of the surfaces $s$ and $b$, as shown in figure 3 . With shallow lateral curvature $1 / \tilde{R} \ll 1$ it is easy to show that the projection of the avalanche edge onto the $(z=0)$-plane is approximately elliptical in shape, with downslope major axis, $r_{b}$, and cross-slope minor axis, $r_{b}\left(1-\left(r-h_{c}\right) /(2 R)\right)=$ $y_{\max }$. The major axis of the cap $\tilde{r}_{b}=32 \mathrm{~cm}$, and the maximum height, $\tilde{h}_{c}=22 \mathrm{~cm}$, occurs at the centre of the cap; which is positioned at $\tilde{x}=6$ and $\tilde{y}=0 \mathrm{~cm}$. The cap is mounted on a metal frame that is pivoted at the top of the chute. The avalanche is released manually by pulling a rope attached to the cap; this raises it rapidly upwards and away from the direction of the avalanche flow about a pivot point. The physical lengths of the chute and the cap geometry are summarized in table 1.

In the experiments the granular material is released from rest; however, this causes 


\begin{tabular}{lcl}
\multicolumn{1}{c}{ Variable } & Symbol & Value \\
Radius of curvature & $R$ & $11(110 \mathrm{~cm})$ \\
Angle of inclination & $\zeta_{0}$ & $40^{\circ}$ \\
End of parabolic section & $x_{a}$ & $17.5(175 \mathrm{~cm})$ \\
Beginning of flat section & $x_{b}$ & $21.5(215 \mathrm{~cm})$ \\
Major axis of cap & $r_{b}$ & $3.2(32 \mathrm{~cm})$ \\
Cap height & $h_{c}$ & $2.2(22 \mathrm{~cm})$ \\
x-position of the cap centre & $x_{0}$ & $0.6(6 \mathrm{~cm})$ \\
y-position of the cap centre & $y_{0}$ & $0.0(0 \mathrm{~cm})$ \\
Non-dimensional time step & $\Delta t$ & 0.01 \\
Downslope diffusion coefficient & $\mu_{x}$ & 0.5 \\
Cross-slope diffusion coefficient & $\mu_{y}$ & 0.1 \\
Number of points & $I$ & 331 \\
Number of elements & $J$ & 600
\end{tabular}

TABLE 1. The non-dimensional chute geometry and the initial conditions for the granular material are summarized, along with the numerical parameters used in the simulations. Equivalent physical lengths are indicated in brackets.

problems in the theory, because the term $\boldsymbol{v} /|\boldsymbol{v}|$ is undefined in the Coulomb basal friction law when $\boldsymbol{v}=\mathbf{0}$. This was present in the original Savage \& Hutter (1989) theory, and there is no simple way to completely eliminate it. In the calculations in this paper it is simply assumed that $\boldsymbol{v} /|\boldsymbol{v}|=\mathbf{0}$ when $\boldsymbol{v}=\mathbf{0}$. That is, it is assumed that there is no basal friction when the avalanche is at rest.

\subsection{Photogrammetric measurements}

A Canon F1 high-speed camera was used to follow the dynamic evolution of the avalanche boundary during each experiment. Typically 10 frames per second were taken with an exposure time of $1 / 1000 \mathrm{~s}$ to minimize blurring of the avalanche. Single image photogrammetric techniques (e.g. Kraus 1982) were used to determine the position of the edge of the flowing avalanche. Although the position of the avalanche edge does not lie in a plane, the shallowness of the superposed geometry allows its position to be determined to within $\pm 1 \mathrm{~cm}$.

Stereo photography allows the three-dimensional position of a point to be reconstructed from two pictures taken from different locations (e.g. Buchholz \& Rüger 1972; Kraus 1982) and at the same instant in time if the object is moving. For the final avalanche deposit the granular material is at rest and the images do not have to be taken simultaneously. A Technical Measurement Camera (TMK 21) belonging to the Institute for Photogrammetry of the Technische Universität in Darmstadt was used to obtain two image pairs. This middle format camera $(9 \times 13 \mathrm{~cm}$ negatives $)$ is specifically designed for surveying purposes and has a built-in Reseau plate to correct for uneveness of the film. A basis of approximately $1.2 \mathrm{~m}$ between the camera positions was used. This yields an excellent three-dimensional image of the avalanche when the image pairs are viewed through a stereoscope. The photographs were evaluated on the AC3 analytical stereo-evaluation device at the Institute of Photogrammetry and used to determine the free-surface height of the stationary deposit of granular material. 

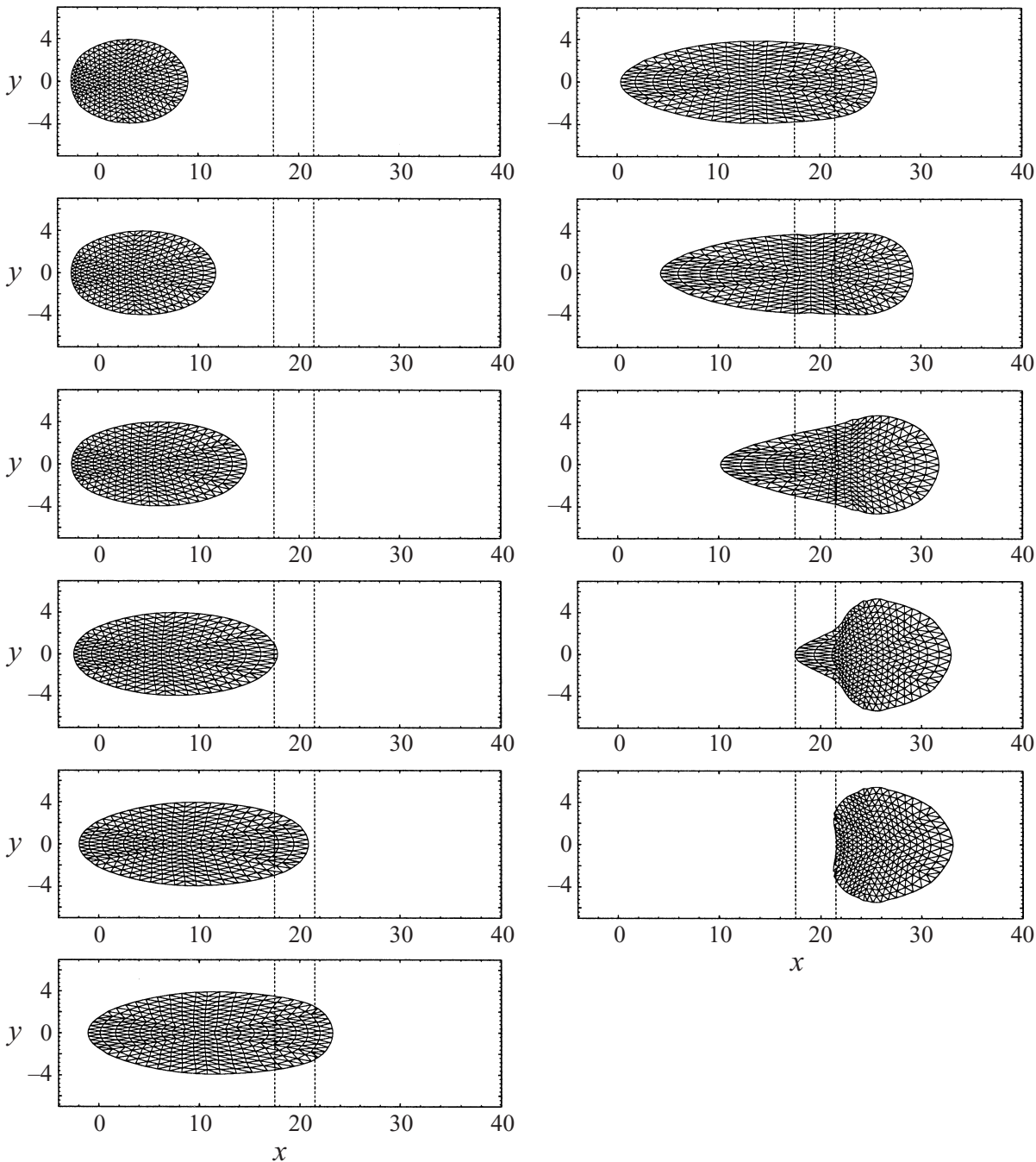

FIGURE 4. The dimensionless displacement and deformations of a ten-ring numerical grid used to simulate the avalanche experiment V05 (vestolen). The image sequence is shown at the same times as those in figures 5 and 7 . The vertical dashed lines at $x=17.5$ and $x=21.5$ indicate the beginning and end of the transition zone, with the $40^{\circ}$ inclined channel to the left and the horizontal run-out plane to the right.

\section{Numerical method}

An explicit in time, spatially two-dimensional, Lagrangian, mixed finite-volume finite-difference scheme is used to solve the depth-integrated equations (2.1)-(2.3). The avalanche is discretized into a finite number of triangular elements, which form a grid that moves and deforms with the motion of the avalanche as figure 4 illustrates. The set of points used to generate this grid reflects the initial experimental configuration of the avalanche, which is approximately elliptical in shape. Details of the discretization process are given in Appendix B.

Assuming that the position, thickness and velocity at the grid nodes are known at time step $k-1$, as well as the initial volume of material in each triangular cell, the numerical algorithm is as follows. 
(i) The new positions of the element corners, $i$, are computed by integrating equations $\mathrm{d} x / \mathrm{d} t=u$ and $\mathrm{d} y / \mathrm{d} t=v$ explicitly forward from time step $k-1$ to $k$ :

$$
x_{i}^{k}=x_{i}^{k-1}+\Delta t u_{i}^{k-1 / 2}, \quad y_{i}^{k}=y_{i}^{k-1}+\Delta t v_{i}^{k-1 / 2},
$$

where $\Delta t$ is the length of the time step.

(ii) The area, $A_{j}^{k}$, of the triangular elements, $j$, at time step $k$ is computed by forming the scalar triple product

$$
A_{j}^{k}=\frac{1}{2}\left|\boldsymbol{k} \cdot\left[\left(\boldsymbol{p}_{\beta_{j}}^{k}-\boldsymbol{p}_{\alpha_{j}}^{k}\right)_{\wedge}\left(\boldsymbol{p}_{\gamma_{j}}^{k}-\boldsymbol{p}_{\alpha_{j}}^{k}\right)\right]\right|,
$$

where $\boldsymbol{p}_{\alpha_{i}}, \boldsymbol{p}_{\beta_{j}}, \boldsymbol{p}_{\gamma_{j}}$ are the position vectors of the three neighbouring points to triangular element $j$, and $\boldsymbol{k}$ is the unit normal to the curvilinear coordinate plane.

(iii) The volume in each material region of the avalanche is conserved (see Appendix C). In particular it is conserved in each triangular element of the avalanche, so its current volume, $V_{j}^{k}$, is equal to $V_{j}^{0}$, the initial volume. It follows that the current thickness

$$
h_{j}^{k}=V_{j}^{0} / A_{j}^{k},
$$

is given by dividing the initial volume by the current area of the the triangular element.

(iv) The new velocity components are then computed by an explicit forward step in time

where the acceleration terms are

$$
\begin{aligned}
& u_{i}^{k+1 / 2}=u_{i}^{k-1 / 2}+\Delta t(\mathrm{~d} u / \mathrm{d} t)_{i}^{k-1 / 2}, \\
& v_{i}^{k+1 / 2}=v_{i}^{k-1 / 2}+\Delta t(\mathrm{~d} v / \mathrm{d} t)_{i}^{k-1 / 2},
\end{aligned}
$$

$$
\begin{aligned}
\left(\frac{\mathrm{d} u}{\mathrm{~d} t}\right)_{i}^{k-1 / 2}= & \sin \zeta_{i}^{k}-\left(u_{i}^{k-1 / 2} /\left|\boldsymbol{v}_{i}^{k-1 / 2}\right|\right) \tan \delta_{i}^{k}\left(\cos \zeta_{i}^{k}+\lambda \kappa_{i}^{k}\left(u_{i}^{k-1 / 2}\right)^{2}\right) \\
& -\epsilon \cos \zeta_{i}^{k}\left(\left(K_{x}\right)_{i}^{k}\left(\frac{\partial s}{\partial x}\right)_{i}^{k}+\left(1-\left(K_{x}\right)_{i}^{k}\right)\left(\frac{\partial b}{\partial x}\right)_{i}^{k}\right)+\left(\Psi_{x}\right)_{i}^{k} \\
\left(\frac{\mathrm{d} v}{\mathrm{~d} t}\right)_{i}^{k-1 / 2}= & -\left(u_{i}^{k-1 / 2} /\left|\boldsymbol{v}_{i}^{k-1 / 2}\right|\right) \tan \delta_{i}^{k}\left(\cos \zeta_{i}^{k}+\lambda \kappa_{i}^{k}\left(u_{i}^{k-1 / 2}\right)^{2}\right) \\
& -\epsilon \cos \zeta_{i}^{k}\left(\left(K_{y}\right)_{i}^{k}\left(\frac{\partial s}{\partial y}\right)_{i}^{k}+\left(1-\left(K_{y}\right)_{i}^{k}\right)\left(\frac{\partial b}{\partial y}\right)_{i}^{k}\right)+\left(\Psi_{y}\right)_{i}^{k} .
\end{aligned}
$$

The variables $\zeta_{i}^{k}, \delta_{i}^{k}, \kappa_{i}^{k},\left(K_{x}\right)_{i}^{k}$ and $\left(K_{y}\right)_{i}^{k}$ are the inclination angle, the basal friction angle, the curvature, and down-slope and cross slope Earth pressures, respectively, at time step $k$ and point $i$. The active and passive states are computed from an algorithm given in Appendix D. The thickness gradients have been rewritten using the identity $h=s-b$, where $s$ is the height of the free surface and $b$ is the height of the basal topography above the $(z=0)$-plane. The discretization of the free-surface gradients $(\partial s / \partial x)_{i}^{k}$ and $(\partial s / \partial y)_{i}^{k}$ at time step $k$ and point $i$ is discussed in Appendix E. The gradients of the basal topography $(\partial b / \partial x)_{i}^{k}$ and $(\partial b / \partial y)_{i}^{k}$ are given explicitly from the prescribed basal geometry. Finally, the terms $\left(\Psi_{x}\right)_{i}^{k}$ and $\left(\Psi_{y}\right)_{i}^{k}$ represent the contribution from artificial viscosity (see Appendix F), which is introduced to diffuse sharp gradients in both the avalanche velocity and thickness when shocks develop 
close to the end of the simulation. This is discussed further in the next Section, but it should be noted that the algorithm is stable provided shocks do not develop.

(v) In the last step the position, thickness and velocity components are updated and the iteration starts with these values from step (i).

The algorithm can be started from rest given an initial thickness distribution $h_{j}^{0}$ and initial grid point positions $\left(x_{i}^{0}, y_{i}^{0}\right)$. This requires the loop to start at position (iv) with an explicit time step of length $\Delta t / 2$ to advance the velocity $\boldsymbol{v}_{i}^{0}=\mathbf{0}$ to $\boldsymbol{v}_{i}^{1 / 2}$. Alternatively, the algorithm can start at position (i) by prescribing an initial velocity field $\boldsymbol{v}_{i}^{-1 / 2}$.

The Lagrangian numerical grid deforms and moves with the avalanche as shown in a typical evolution of the numerical grid in figure 4 . Initially the grid is approximately elliptical in shape with the major axis oriented in the downslope direction. As the avalanche flows downhill it stretches out and the triangular grid cells gradually become obtuse. This can reduce the accuracy of the derivative approximations if the angles become too large. For the simulations presented in this paper the error is small. However, on longer chutes where the uniaxial extension is more prolonged it may be necessary to compute a new grid whenever the triangular elements become too obtuse. As the avalanche passes through the transition zone onto the horizontal run-out plane it compresses in the downslope direction and spreads out laterally. It follows that the triangular elements once again become acute angled in the final configuration of the numerical grid.

\section{Comparison of experiments with theoretical predictions}

In this section the measurements from three laboratory experiments are presented and compared with the results of numerical simulations. The system of equations (2.1)-(2.3) contain two non-dimensional parameters, $\epsilon$ and $\lambda$, associated with the geometry of the avalanching material and the chute. Provided the geometry is similar these equations predict the same avalanche flow irrespective of whether it is in a smallscale laboratory experiment or in a large-scale mountain environment. The results of both the experiments and the numerical computations are therefore presented in non-dimensional variables to achieve as much generality as possible. The appropriate physical variables for a particular application can then be constructed by applying the scaling (2.4). A non-dimensionalization is chosen in which the length scale in the $x, y$-directions is the same as in the $z$-direction. That is, $L=10 \mathrm{~cm}, H=10 \mathrm{~cm}$ and $\mathscr{R}=10 \mathrm{~cm}$ giving $\epsilon=H / L=1$ and $\lambda=L / \mathscr{R}=1$. This scaling makes it easier to interpret the results as the aspect ratio of the physical avalanche is preserved. The physical and non-dimensional geometry of the chute is summarized in table 1.

Two material parameters are required by the theory, the bed friction angle, $\delta$, and the internal angle of friction, $\phi$. Hutter \& Koch (1991) showed that the avalanche motion is relatively insensitive to changes in the internal angle of friction. It is therefore not essential to measure it to a high degree of accuracy and it is simply assumed that it is equal to the angle of repose of a conical pile of the granular material built up on a horizontal frictional plane. Measuring the bed friction angle is more difficult. Experiments suggest that there is a range of bed friction angles, which may differ by up to $4^{\circ}$ (Hungr \& Morgenstern 1984a,b), depending on whether the motion is static or dynamic. Savage \& Hutter (1989) and Greve \& Hutter (1993) measured the static $\delta$ by tilting a planar section of the bed topography and measuring the angle at which a stationary sample of the granular material began to slide. In their 
simulations they used a dynamic bed friction angle, which they computed by reducing the measured static angle by $4^{\circ}$ following Hungr \& Morgenstern $(1984 a, b)$. Very good agreement between theory and experiment was obtained in both one-dimensional and unconfined two-dimensional cases by using this value.

The physical mechanisms responsible for the $10-15 \%$ difference between static and dynamic bed friction angles are not well understood. However, the dilatant nature of granular materials when sheared (Reynolds 1885) and the granular temperature induced by inter-particle collisions in rapid flows (Jenkins \& Savage 1983) are probably the prime mechanisms. The predictive capability of the Savage-Hutter model is of course reduced by the deviations from the Coulomb basal dry friction law. For practical purposes, however, a simple parameter study using different values of $\delta$ is enough to provide upper and lower bounds for the avalanche motion that are sufficiently close together to be of great practical use in hazard forecasting. In future our understanding of the tribology of the rapidly moving and evolving interface between the base of the avalanche and the chute is likely to advance considerably, and, when more accurate basal friction laws become available they can easily be incorporated into the structure of the Savage-Hutter theory.

In the experiments presented in this paper it was found that at the avalanche front, where high velocities are attained, the grains enter into a rapid collisional flow regime (Jenkins \& Savage 1983) that fluidizes the basal layer. It is postulated that this can reduce the basal friction and it therefore is a plausible explanation for long run-out distances attained by very large-mass avalanches. However, in our experiments the grains in the collisional regime experienced more basal drag than those which flow more slowly. Thus, when a constant dynamic bed friction angle $\delta_{0}$ was used to simulate the flow it was always found that the front was modelled well but the tail moved too slowly. In order to quantify this effect a variable bed friction angle has been introduced that is linearly reduced from the dynamic value of the front in the rear three-quarters of the avalanche:

$$
\delta= \begin{cases}\delta_{0}, & x \geqslant x_{f}-\left(x_{f}-x_{r}\right) / 4 \\ \delta_{0}-m_{\delta}\left(x_{f}-\left(x_{f}-x_{r}\right) / 4-x\right), & x<x_{f}-\left(x_{f}-x_{r}\right) / 4 .\end{cases}
$$

where $m_{\delta}=1$ is the bed friction reduction factor and, $x_{f}$ and $x_{r}$ are the position of the front and rear of the avalanche, respectively. Gray et al. (1999) have presented a comparison of the numerical simulations with and without this bed friction reduction to show the effect that (5.1) has on the computations. All our experiment simulations were in very good agreement with measurements when the same law was used. While (5.1) is only an empirical relation there are a number of competing physical mechanisms that may be responsible for it. The rapid collisional flow regime can fluidize the particles near the chute topography and introduce a rate dependence that can either increase or decrease the drag depending on the mass of the avalanche. Topographical effects may add to this as well as lubrication and damage effects on the chute itself. A considerable research effort is required before such physical effects can be properly measured and quantitatively understood. Only then will it be possible to fully explain the final $10-15 \%$ difference in the dynamic and static bed friction angles.

\subsection{Experiment V05: Vestolen plastic beads}

Experiment V05 was performed with rounded plastic beads (Vestolen) with a mean diameter of $2-3.5 \mathrm{~mm}$, basal angle of friction $\delta=27^{\circ}$ and internal angle of friction $\phi=33^{\circ}$. A sequence of photographs showing the evolution of the avalanche in the 

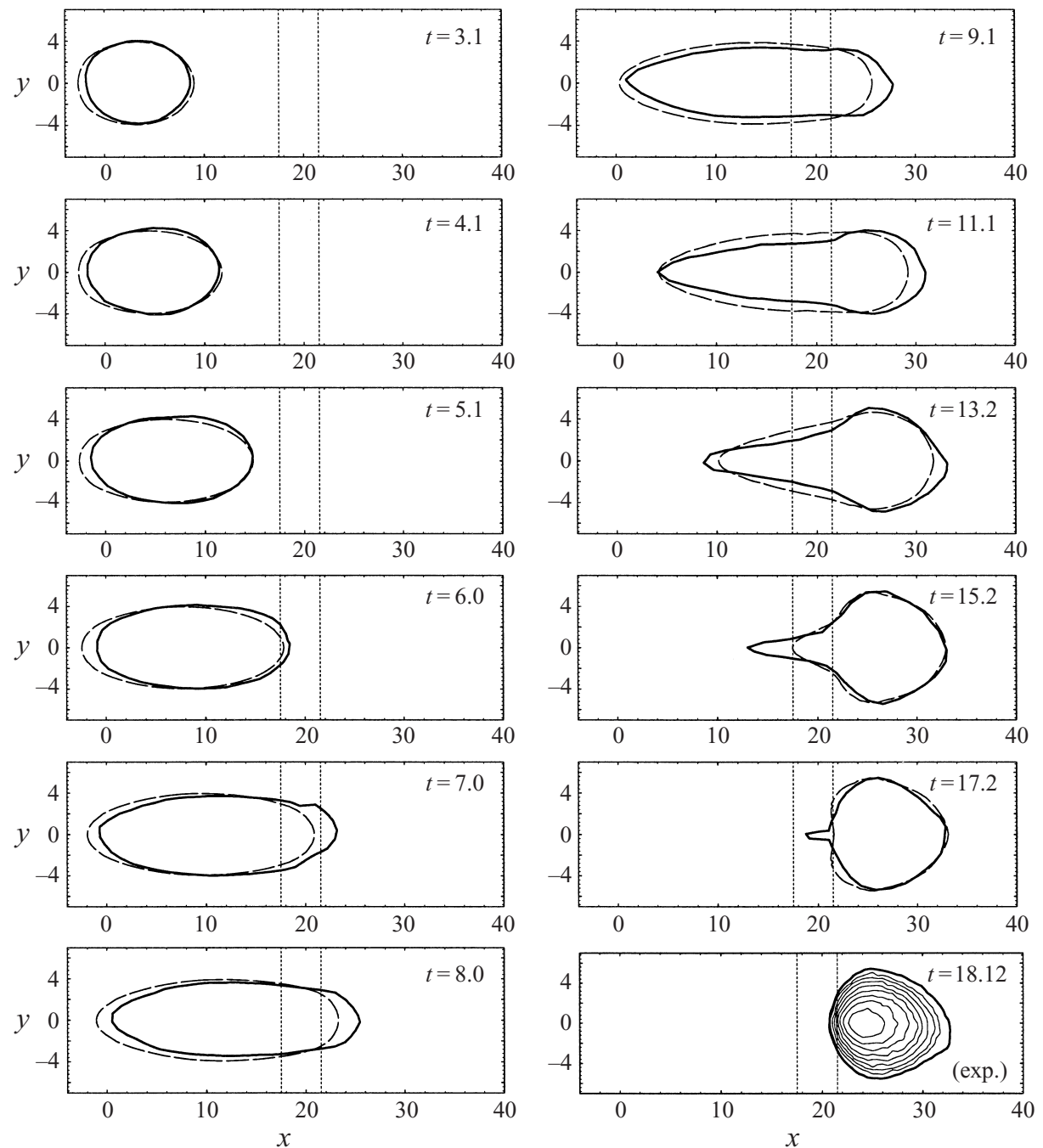

FIGURE 5. A comparison between the dimensionless actual avalanche (solid) boundary in experiment V05 (vestolen) and the computed (dashed) edge is plotted at a sequence of time steps in projected curvilinear coordinates $(x, y)$. The vertical dashed lines at $x=17.5$ and $x=21.5$ indicate the position of the transition zone, with the $40^{\circ}$ inclined channel to the left and the horizontal run-out plane to the right. In the bottom right panel the thickness distribution of the experimental avalanche is illustrated using 0.1 unit contours.

experiment is shown in figure 2. This evolution is typical of all three experiments described in this paper. The granular material is released from the cap on the inclined section of the chute and rapidly spreads out in the downhill direction, so that when the avalanche front reaches the run-out plane the tail has barely moved from its initial position. As the avalanche flows through the transition zone the lateral confinement ceases and the granular material spreads out laterally. This produces a very strong nose and tail structure, in which the nose is spreading and the tail is being channelized by the topography.

A single-image photogrammetric method has been used to extract the position of the avalanche boundary from a series of these photographs. A comparison of the 

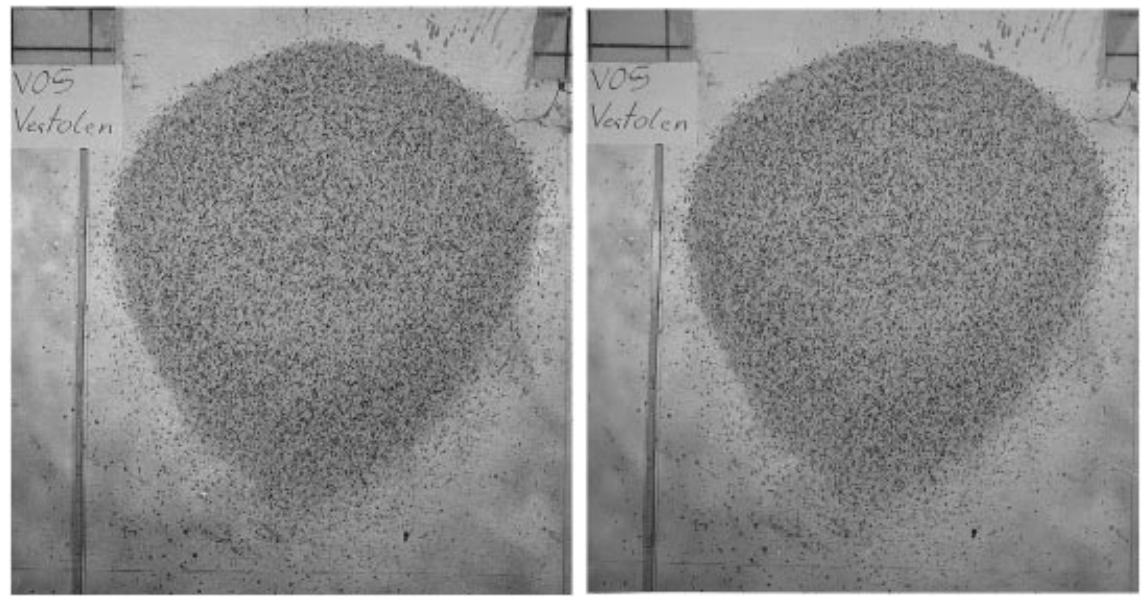

FIGURE 6. An image pair of the final avalanche deposit on the horizontal run-out plane in experiment V05. The transition zone and parabolic section of the chute is along the top edge of the pictures and the downslope direction is from top to bottom. A three-dimensional image of the avalanche can be seen with the use of a stereoscope. It is also possible to view the three-dimensional image without the use of a stereoscope after some practice. The trick is to hold the page at arms length and focus in the far distance, so that one sees double. By varying the distance of the page from the eye it is then possible to overlap the left and right images so that the brain can interpret it in three dimensions.

measured (solid) and computed (dotted) boundary for a sequence of time steps is illustrated in figure 5. In each panel the downslope curvilinear coordinate runs along the $x$-axis and the cross-slope coordinate lies along the $y$-axis. The symmetry line of the parabolic channel runs along the line $y=0$; this also represents the set of points with the minimum height above the curvilinear surface $z=0$, or the Talweg. The fine dotted lines indicate the end of the parabolic channel at $x_{a}=17.5$ and the beginning of the plane horizontal section of the chute at $x_{b}=21.5$.

In the final panel of figure 5, isolines of the measured thickness are plotted using equally spaced contour intervals of 0.1 non-dimensional units $(1 \mathrm{~cm}$ physical) above the edge, where the height is zero. These contours were determined by a stereo photogrammetric method from the two photographs in figure 6. In figure 7 the computed thickness is illustrated at the same time steps as in figure 5, and the bottom right panel shows a comparison with the final experimental thickness distribution.

The dominant deformation in the parabolic channel is due to downslope stretching of the avalanche, in which the front section moves much faster than the rear. In response to this deformation field the thickness of the avalanche is reduced throughout the avalanche. At $t=5.1$ the maximum value lies between 0.8-0.9 compared with the initial value $h_{c}=2.2$. The tendency for the avalanche to spread in the cross-slope direction in response to the Earth pressure terms, observed on plane slopes (Koch et al. 1994; Gray et al. 1999) is not observed here. Instead the channelizing effect of the parabolic basal topography is almost in exact balance with the cross-slope spreading effect of the Earth pressure terms. Initially there is a small cross-slope divergence of the avalanche while the thickness gradient, $\partial h / \partial y$, is relatively large, but as the thickness decreases due to the downslope stretching the lateral gradients decrease and the channelizing effect of the topography, $\partial b / \partial y$, dominates. The general position and shape of the experimental and computed avalanche agree extremely well. In particular the front of the computed avalanche is in almost exact agreement with the experiment. 

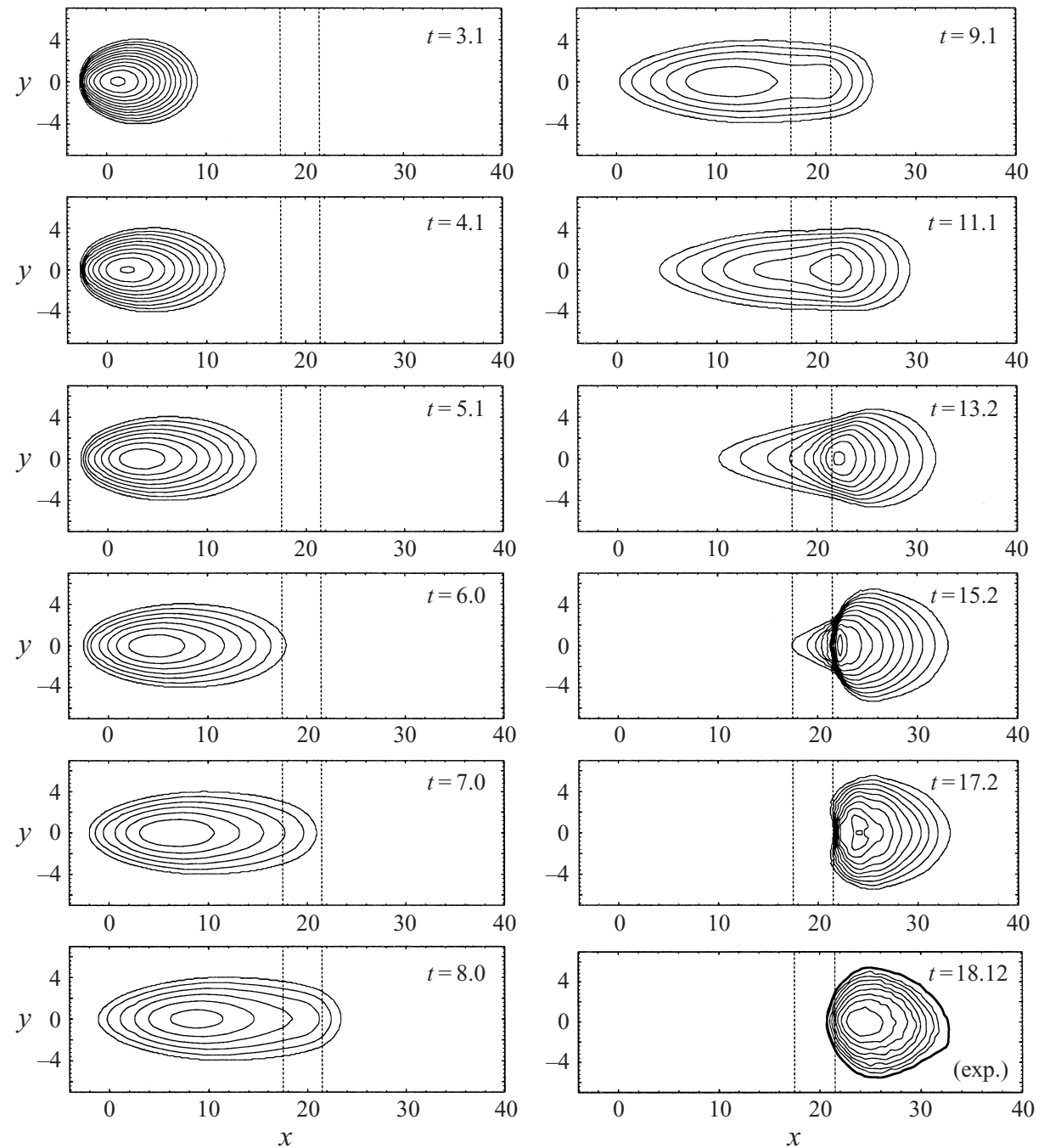

FIGURE 7. The computed dimensionless avalanche thickness for experiment V05 (vestolen) is illustrated at the same times as in figure 5, using 0.1 unit contour intervals. The bottom right panel shows the final experimental thickness distribution, which agrees very well with that predicted (immediately above).

As the front, or nose, of the avalanche reaches the run-out plane, at $t=9.1$, it decelerates rapidly as the gravity forcing ceases and the basal friction dissipates the accumulated energy. The avalanche spans all three sections of the chute. In the channel section the tail of the avalanche continues to stretch in the downslope direction reducing in thickness correspondingly. However, in the transition zone $\left(x_{a} \leqslant x \leqslant x_{b}\right)$ the avalanche is dominated by downslope convergence, which increases the thickness. On the horizontal plane the partial confinement of the concave parabolic section ceases and there is cross-slope motion of the avalanche.

The avalanche develops a 'tadpole' like nose-and-tail structure at $t=15.2$, which is characteristic of all the experiments performed on this chute. This shape is due to the change from parabolic lateral confinement on the chute to unconfined flow on the run-out plane. In the nose, where the avalanche has almost come to rest, 

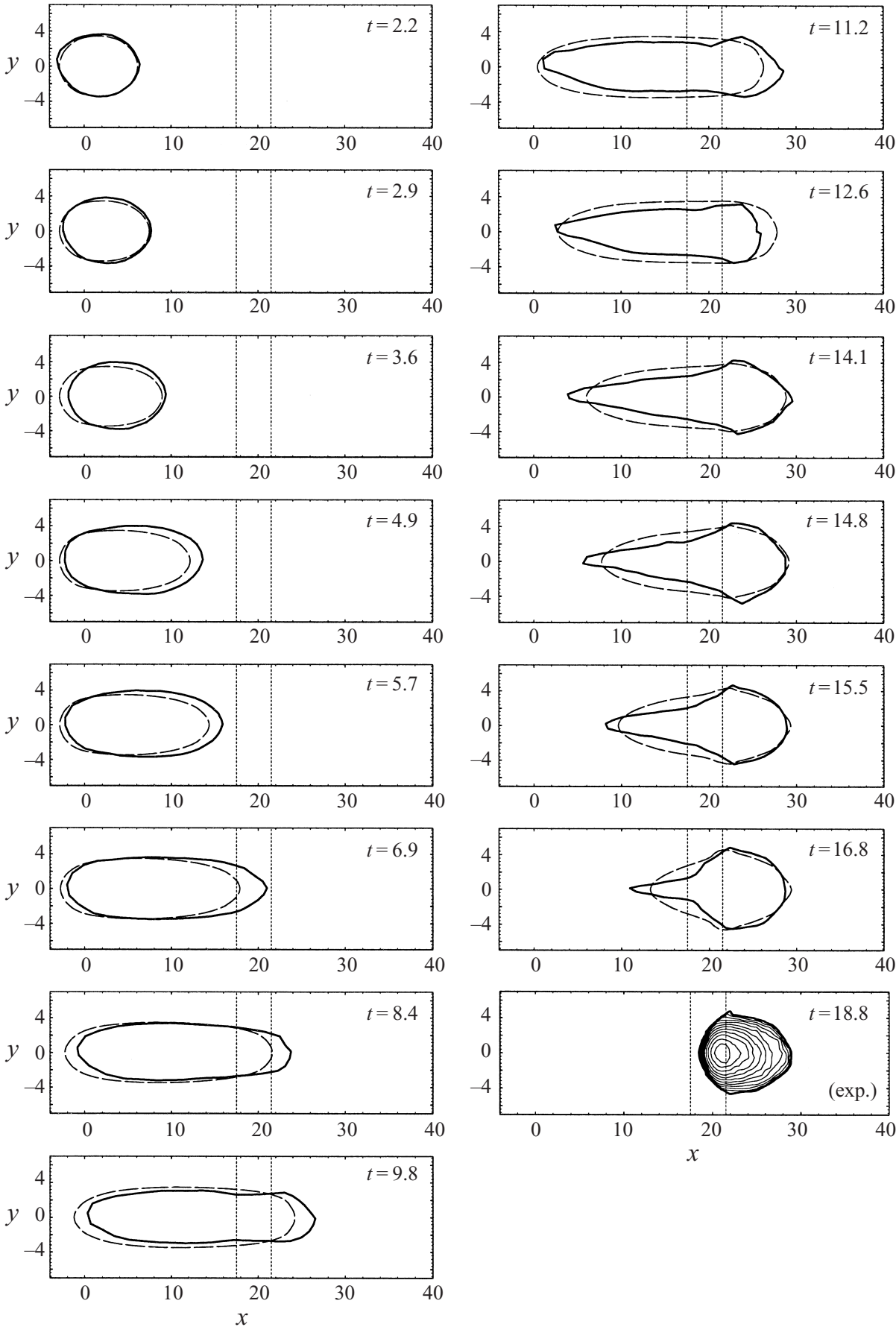

FIGURE 8. A comparison between the dimensionless actual avalanche (solid) boundary in experiment V04 (marble) and the computed (dashed) edge is plotted for a sequence of time steps in projected curvilinear coordinates $(x, y)$. In the bottom right panel the thickness distribution of the experimental avalanche is illustrated using 0.1 unit contours. 


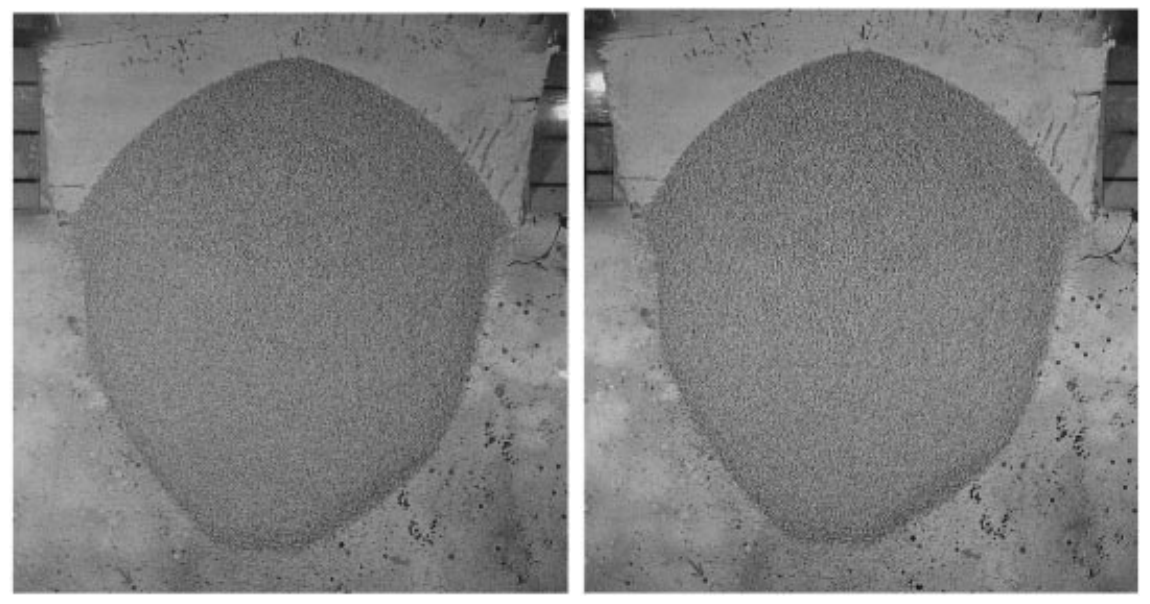

FIGURE 9. An image pair of the final avalanche deposit on the horizontal run-out plane in experiment V04. The transition zone and parabolic section of the chute is along the top edge of the pictures and the downslope direction is from top to bottom. A three-dimensional image of the avalanche can be seen with the use of a stereoscope or the trick described in figure 6 .

the agreement is excellent. However, in the tail section, which is still being forced by the gravitational acceleration, the avalanche is channelized somewhat more than predicted. At the interface between the nose and the tail very sharp gradients in thickness and velocity develop as shown in figure 9 at $t=15.2$. This is in fact a granular shock wave (Gray \& Hutter 1997, 1998), which propagates upslope. The granular material flowing in the tail has typical physical velocities of $1-2 \mathrm{~m} \mathrm{~s}^{-1}$ and as it passes through the shock wave it increases in thickness and comes to rest. The formation and propagation of this shock wave in the final three non-dimensional time units presents significant difficulties for the numerical method as it is not shock capturing. In the experiments in this paper only weak diffuse shocks develop and reasonable simulations can be performed by introducing some artificial numerical viscosity into the momentum equations (4.6) and (4.7). These are only turned on after the tenth non-dimensional time unit.

The computed avalanche comes to rest at $t=17.2$, whilst the last particles come to rest at $t=18.2$ in the experiment. This difference is due to a very thin slow moving tail that can be seen in the penultimate panel of figure 5. This thin tail is not resolved in the numerical simulation. The position and thickness of the computed and experimental granular deposits are easily compared in the last two panels of figure 9. The computed position of the boundary, the maximum thickness and the position of the maximum thickness are all in excellent agreement.

\subsection{Experiment V04: Marble chips}

In experiment V04 marble chips with a mean diameter of $2-4 \mathrm{~mm}$ were used. These have quite a pointed geometry and their surface is considerably rougher than the plastic beads. As a result there is considerably more interparticle and basal friction, which is reflected in the higher internal, $\phi=43^{\circ}$, and basal, $\delta=33^{\circ}$, friction angles.

In a point-mass model, in which the thickness gradients are ignored, the dominant balance in the downslope momentum balance (2.2) is between the acceleration, gravity and basal drag. It follows that on the inclined section of the chute the downslope acceleration is proportional to $\sin \zeta_{0}-\cos \zeta_{0} \tan \delta$. An increase in the bed friction 

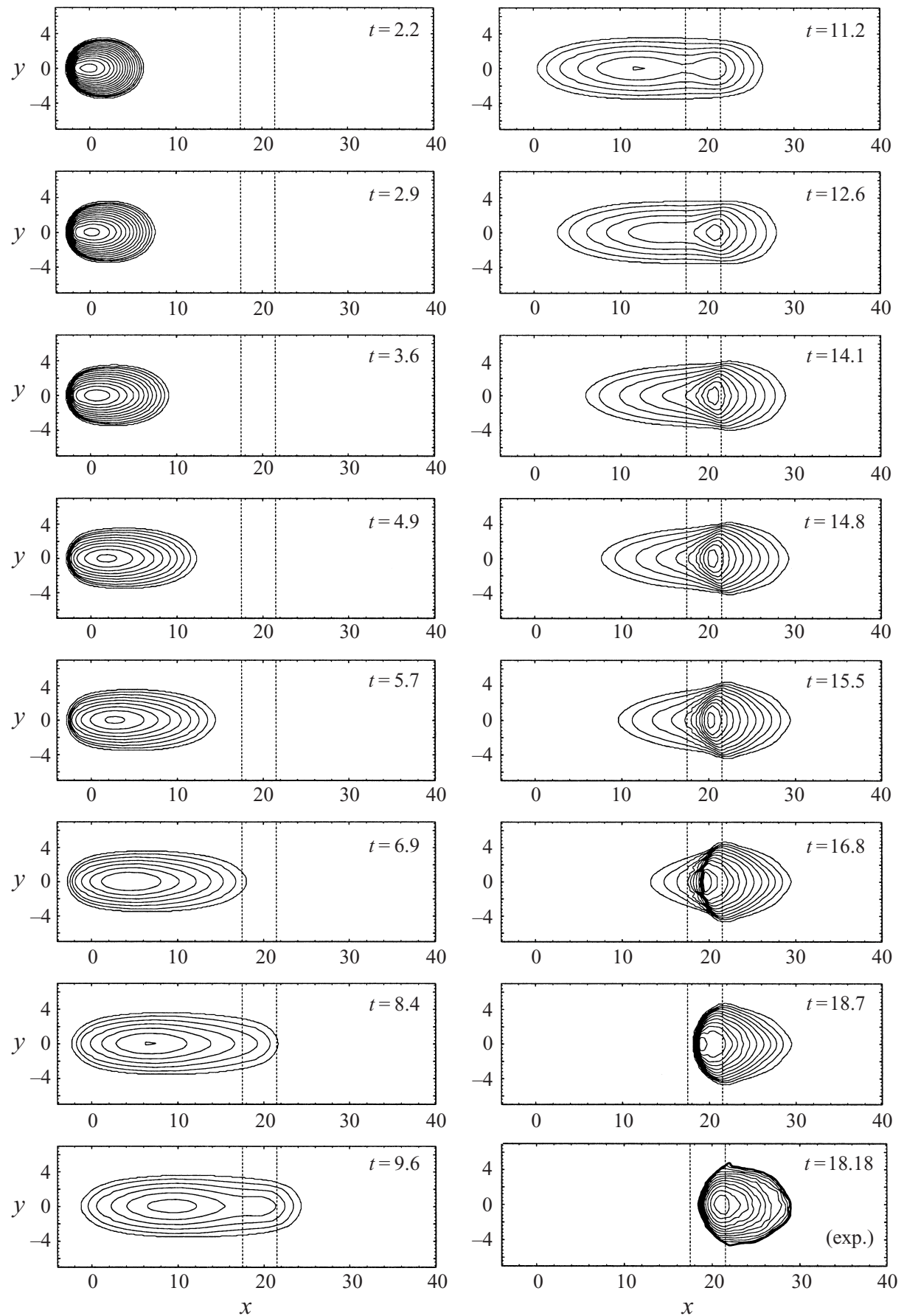

FIGURE 10. The computed dimensionless avalanche thickness for experiment V04 (marble) is illustrated at the same times as in figure 8 , using 0.1 unit contour intervals. The final experimental thickness distribution is shown bottom right. 

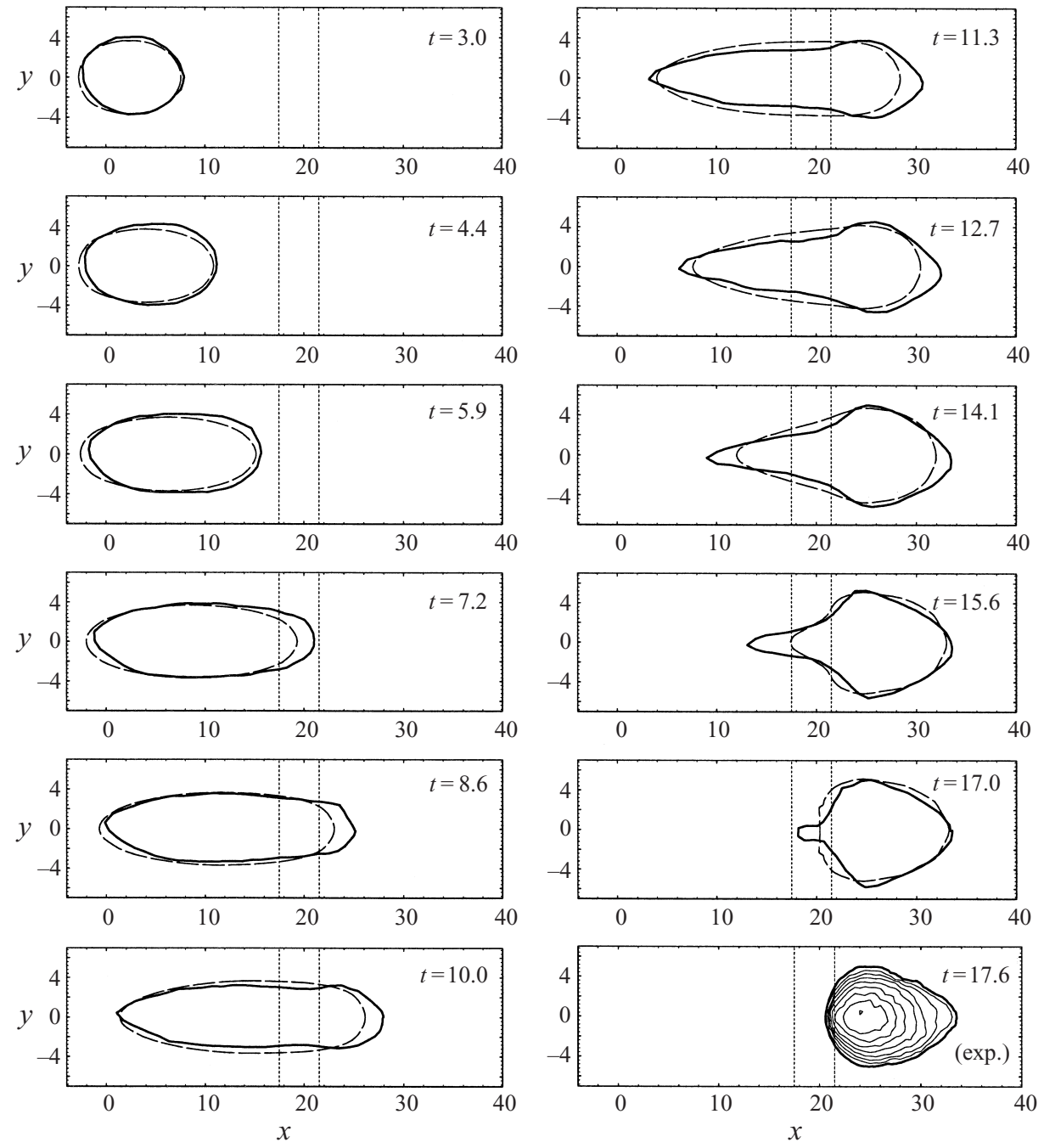

FIGURE 11. A comparison between the dimensionless actual avalanche (solid) boundary in experiment V03 (quartz) and the computed (dashed) edge is plotted at a sequence of time steps in projected curvilinear coordinates $(x, y)$. In the bottom right panel the thickness distribution of the experimental avalanche is illustrated using 0.1 unit contours.

angle therefore implies that the avalanche accelerates more slowly and reaches lower velocities on the inclined section of the chute. Furthermore, on the horizontal plane, where $\zeta=0$, the acceleration is proportional to $-\tan \delta$ and the avalanche decelerates more rapidly. It is therefore expected that the avalanche will not travel as far in experiment V04 as in V05.

A comparison of the measured (solid) and computed (dashed) avalanche boundary is illustrated for a sequence of time steps in figure 8 . The overall evolution of the shape and thickness distribution (figure 7) of the avalanche as it flows down the chute is broadly the same as in experiment V05. However, there are a number of important differences, which are due to the increased bed friction angle. As expected the avalanche does not travel as far as in experiment V05. The position of the centre of 

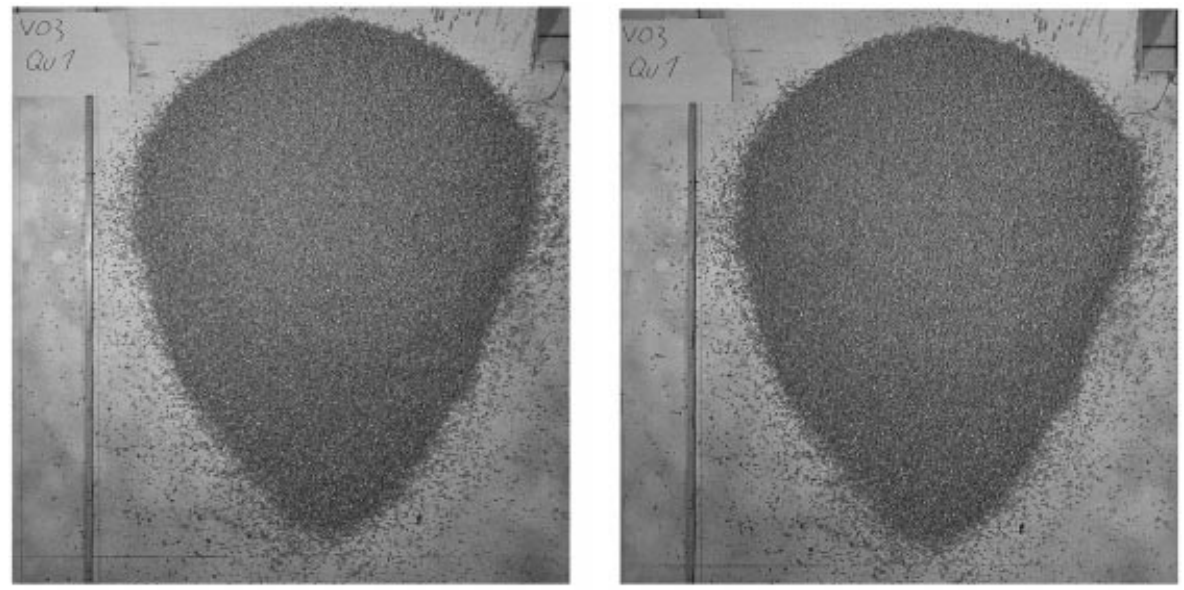

FIGURE 12. An image pair of the final avalanche deposit on the horizontal run-out plane in experiment V03. The transition zone and parabolic section of the chute is along the top edge of the pictures and the downslope direction is from top to bottom. A three-dimensional image of the avalanche can be seen with the use of a stereoscope or the trick described in figure 6 .

mass and the maximum run-out distance are approximately 3 and 4 non-dimensional units further upslope than in experiment V05, respectively. Furthermore, even though the avalanche does not travel as far it takes nearly one non-dimensional time unit longer to come to rest. This is also reflected in the velocity magnitudes which are smaller than in experiment V05.

The fact that the avalanche does not run-out as far as V05 implies that much of the material is brought to rest in the transition zone, where the the basal topography still weakly accelerates the flow. The strength of the shock wave that forms at the interface between the nose and tail is correspondingly larger. The evolution of steepening and propagation of the diffuse shock can be seen very well in the thickness contours for time steps $t=14.1,14.8,15.5$ and 16.8 time units in figure 10. It forms at the front of the transition zone and slowly propagates upslope and steepens as the material flowing into it from the tail becomes thinner and thinner.

A balance between the Coulomb basal friction and the active pressure gradient terms in (2.2) suggests that the steepest slope that can arise, $\max (\partial h / \partial x)$, in the rest state is equal to $\tan \delta / K_{x_{a c t}}$. In experiment V04 $\max (\partial h / \partial x)=0.714$, whereas in experiment V05 it is 0.577 , and the slope at the rear of the avalanche is therefore considerably steeper with the contours packed tightly together. This is shown clearly in the final thickness distribution in the computed and experimental avalanche in figure 10 , as well as in the stereo image pair in figure 9 .

\subsubsection{Experiment V03: Quartz1}

Experiment V03 uses quartz chips of mean diameter of 4-5 $\mathrm{mm}$. The internal angle of friction $\phi=39^{\circ}$ and the bed friction angle $\delta=28^{\circ}$. The bed friction angle is only one degree more than in experiment V05 so the point-mass speed of the two avalanches are very close. The two granular materials have quite different internal angles of friction and a comparison of experiments V03 and V05 allows the sensitivity of the flow to changes in $\phi$ to be investigated.

The influence of $\phi$ enters through the Earth pressure coefficients defined in (2.7) and (2.8). The value of the active Earth pressure coefficient determines the amount of 

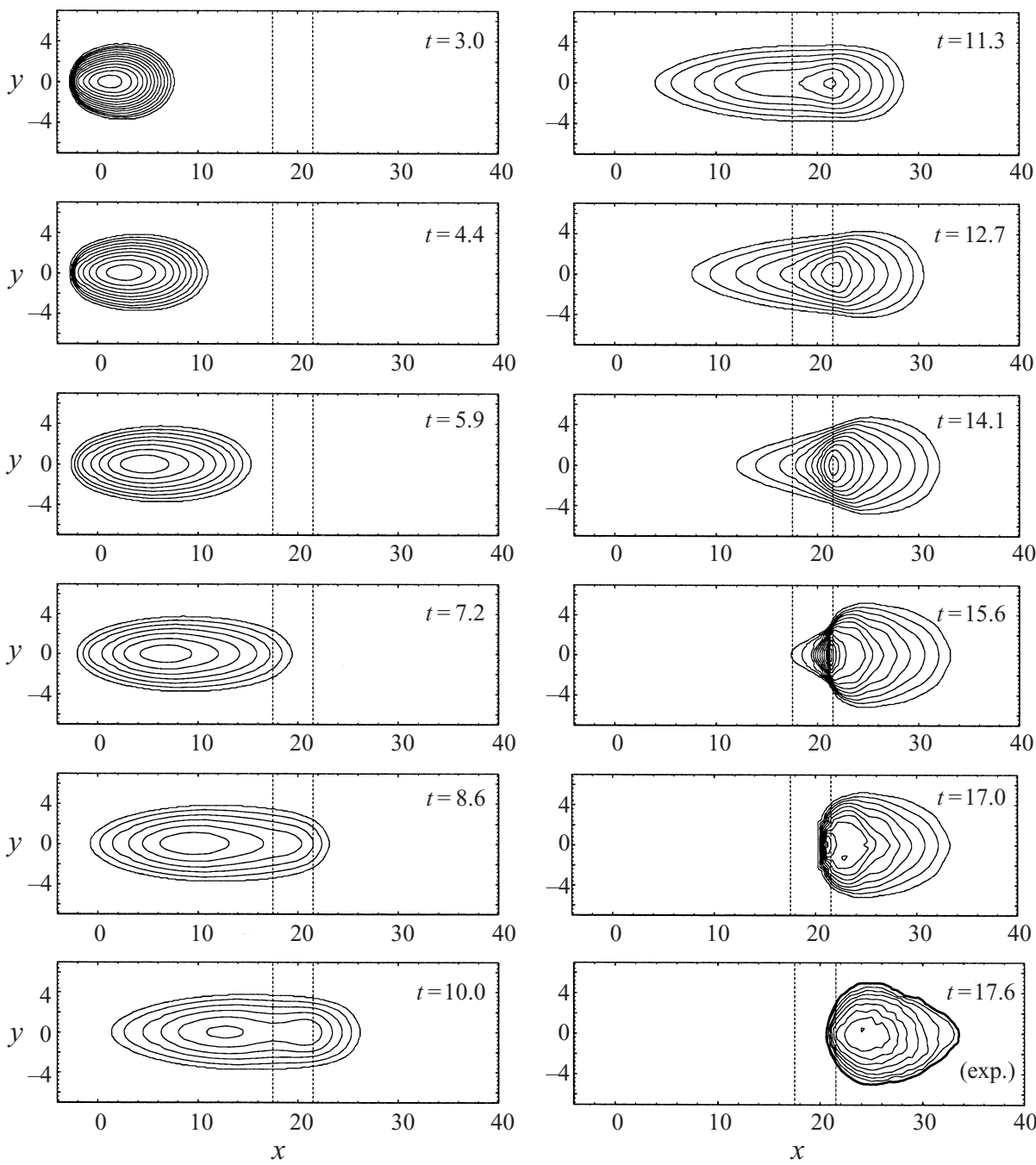

FIGURE 13. The computed dimensionless avalanche thickness for experiment V03 (quartz) is illustrated at the same times as in figure 11 , using 0.1 unit contour intervals. The final experimental thickness distribution is shown bottom right.

spreading in the divergent phase of the avalanche motion and is relatively insensitive to changes in $\phi$. For experiment V03 $K_{x_{a c t}}=0.74$ whilst for experiment V05 $K_{x_{\text {act }}}=0.88$. Both values are close to unity, which implies that the internal stresses are close to isotropic. A direct comparison of the evolution of the avalanche boundary, in figures 11 and 5, and the avalanche thickness, in figures 13 and 7, shows that experiments V03 and V05 are indeed very similar. A balance between the Coulomb basal friction and the active pressure gradient terms in (2.2) once again suggests that maximum inclination of the slopes at the back of the avalanche deposit are slightly larger in experiment V03 than in experiment V05, which the image pairs figures 12 and 6 confirm.

The convergent (passive) state $K_{x_{p a s}}$ is more sensitive to changes in the material parameters. However, it is only activated for a relatively short period of time as the avalanche passes through the transition zone onto the run-out plane, and therefore 
does not have a large influence on the position and shape of the final deposit. In experiment V03 $K_{x_{\text {pas }}}=3.88$, which implies that the downslope normal stress is nearly four times larger than the hydrostatic pressure, whereas in experiment V05 $\phi=33^{\circ}$ implies $K_{x_{p a s}}=2.80$. The shocks at the back of the avalanche occur during rapid maintained convergence and the value of $K_{x_{p a s}}$ will have a significant effect on the shock speed and the avalanche thickness and velocity jumps at such singular surfaces.

\section{Conclusions}

Three laboratory experiments were performed on a chute with complex topography, which demonstrate very good agreement with numerical simulations using a threedimensional extension of the Savage-Hutter avalanche theory (Gray et al. 1999). The experiments show that partial confinement of the avalanche, in the inclined parabolic section of the chute, prevents lateral spreading and strongly channelizes the flow. As the nose of the avalanche flows out onto the unconfined run-out plane it spreads laterally, whilst the tail is still channelized. These topographic effects result in the avalanche developing a tadpole-like shape during the latter stages of the motion. The numerical simulations have been able to reproduce all these features. They therefore provide a tool to predict the motion of avalanches over realistic site-specific topography.

When the bed friction angle, $\delta$, is increased the avalanche accelerates more slowly and attains lower speeds on the inclined chute. It also comes to rest more rapidly on the run-out plane. The maximum run-out distance is therefore decreased and the overall position of the granular deposit is further upslope even though the avalanche takes longer to come to rest. In all the experiments steep gradients in the avalanche and thickness were observed when the granular material came to rest close to or in the transition zone. The formation of the shocks caused significant problems for the numerical method, which is not shock capturing. However, in these experiments the shock was diffuse enough that the inclusion of some artificial viscosity was sufficient to allow the computations to proceed until the avalanche came to rest.

This research was supported by the Deutsche Forschungsgemeinschaft through the SFB 298 project "Deformation und Versagen bei metallischen und granularen Strukturen". We gratefully acknowledge the invaluable assistance of Dr R. Düppe (Institut für Photogrammetrie at the Technische Hochschule Darmstadt) and the help of $\mathrm{H}$. Wall (Labor Mechanik) in constructing the chutes and performing the experiments.

\section{Appendix A. Mapping to rectangular coordinates}

The curvilinear coordinates can be related to a Cartesian coordinate system $O^{\prime} X Y Z$ with basis vectors $\boldsymbol{i}, \boldsymbol{j}, \boldsymbol{k}$ along the $X$-, $Y$-, $Z$-axes, respectively. The $Z$-axis is assumed parallel (but opposite in direction) to the gravitational acceleration and the $Y$-axis is parallel to the $y$-axis. Given curvilinear unit basis vectors $\boldsymbol{g}_{1}, \boldsymbol{g}_{2}, \boldsymbol{g}_{3}$, along the $x$-, $y$-, $z$-axes, respectively, these are related to the Cartesian basis vectors as follows

$$
\left.\begin{array}{l}
\boldsymbol{g}_{1}^{*}=\cos \zeta \boldsymbol{i}-\sin \zeta \boldsymbol{k}, \\
\boldsymbol{g}_{2}^{*}=\boldsymbol{j}, \\
\boldsymbol{g}_{3}^{*}=\sin \zeta \boldsymbol{i}+\cos \zeta \boldsymbol{k} .
\end{array}\right\}
$$


A position vector $\boldsymbol{r}$ can be decomposed into the sum

$$
\boldsymbol{r}=\boldsymbol{r}^{r}+z \boldsymbol{g}_{3}^{*}
$$

where $\boldsymbol{r}^{r}(x, y)$ is a position vector of a point on the curvilinear reference surface $z=0$. The Cartesian components of $\boldsymbol{r}^{r}=X^{r} \boldsymbol{i}+Z^{r} \boldsymbol{k}$ are constructed by solving the integrals

$$
X^{r}=\int_{0}^{x} \cos \zeta\left(x^{\prime}\right) \mathrm{d} x^{\prime}, \quad Z^{r}=Z_{0}-\int_{0}^{x} \sin \zeta\left(x^{\prime}\right) \mathrm{d} x^{\prime},
$$

where $Z_{0}$ is a constant of integration that allows the origins $O$ and $O^{\prime}$ to have different $Z$ positions. Substituting (3.1) into (A 3) the Cartesian components of the curvilinear surface $z=0$ are

$$
\begin{aligned}
& X^{r}= \begin{cases}x \cos \zeta_{0}, & x<x_{a} \\
x_{a} \cos \zeta_{0}-\left(x_{b}-x_{a}\right)\left(\sin \zeta-\sin \zeta_{0}\right) / \zeta_{0}, & x_{a} \leqslant x \leqslant x_{b} \\
x_{a} \cos \zeta_{0}+\left(x_{b}-x_{a}\right)\left(\sin \zeta_{0}\right) / \zeta_{0}+\left(x-x_{b}\right), & x_{b}<x\end{cases} \\
& Z^{r}= \begin{cases}\left(x_{a}-x\right) \sin \zeta_{0}+\left(x_{b}-x_{a}\right)\left(1-\cos \zeta_{0}\right) / \zeta_{0}, & x<x_{a} \\
\left(x_{b}-x_{a}\right)(1-\cos \zeta) / \zeta_{0}, & x_{a} \leqslant x \leqslant x_{b} \\
0, & x_{b}<x\end{cases}
\end{aligned}
$$

Note that the curvilinear coordinate system defined above has a singularity at $z=$ $\left(x_{b}-x_{a}\right) / \zeta_{0}$ for all points $(x, y, z)$ in the range $x_{a} \leqslant x \leqslant x_{b}$. This corresponds to the points at which the lines of constant $(x, y)$ intersect at the centre of the circle used to generate the cylindrical transition zone. Provided the avalanche remains shallow enough to avoid the singularity, this does not present a problem.

\section{Appendix B. Discretization}

The avalanche is discretized into a finite number of triangular elements, which deform with the body. The initial configuration is elliptical in shape, with low eccentricity. This allows the discretization to be performed for a circle and then mapped back to the ellipse.

Consider a circular region $B=\left\{(x, y) \mid x^{2}+y^{2} \leqslant r_{b}^{2} x, y \in R\right\}$ of radius $r_{b}$. A set of points $P \subseteq B$ is constructed with a good resolution of the boundary $\partial B$ and a relatively even distribution of the elements in $P$. The set $P$ is equal to $P_{1} \cup P_{2}$ where

$$
\begin{gathered}
P_{1}=\{(x, y) \mid x=0, y=0\}, \\
P_{2}=\left\{(x, y) \mid x_{i j}=i \frac{r_{b}}{n} \cos \left(\frac{2 \pi j}{m i}\right), y_{i j}=i \frac{r_{b}}{n} \sin \left(\frac{2 \pi j}{m i}\right), \quad \begin{array}{l}
i=1, \ldots, n \\
j=1, \ldots, m i
\end{array}\right\},
\end{gathered}
$$

for integers $m, n$ with $m \geqslant 3$. The set $P_{1}$ consists of a single point at the origin of the circle. The set $P_{2}$ is formed from a series of points on $n$ evenly spaced concentric rings centred on the origin. The first ring has $m$ points whilst the outermost ring, which coincides with the boundary of the circle, has $m n$ points. For ease of notation each point in $P$ is given an index number belonging to the set $L=\{1, \ldots, I\}$, where $I=1+m n(n+1) / 2$ is the total number of points in $P=\left\{\boldsymbol{p}_{i}=\left(x_{i}, y_{i}\right) i \in L\right\}$.

To select the optimal global triangulation of the set of points $P$ a Voronoi Tiling (e.g. Hoschek \& Lasser 1992) is constructed in the $(x, y)$-plane. For a total number of $I$ points, $I$ tiles $F_{i}$ are constructed, which cover the entire $(x, y)$-plane. These are defined by

$$
F_{i}=\left\{\boldsymbol{x} \in R^{2} \mid d\left(\boldsymbol{x}, \boldsymbol{p}_{i}\right) \leqslant d\left(\boldsymbol{x}, \boldsymbol{p}_{j}\right) \forall i \neq j\right\},
$$




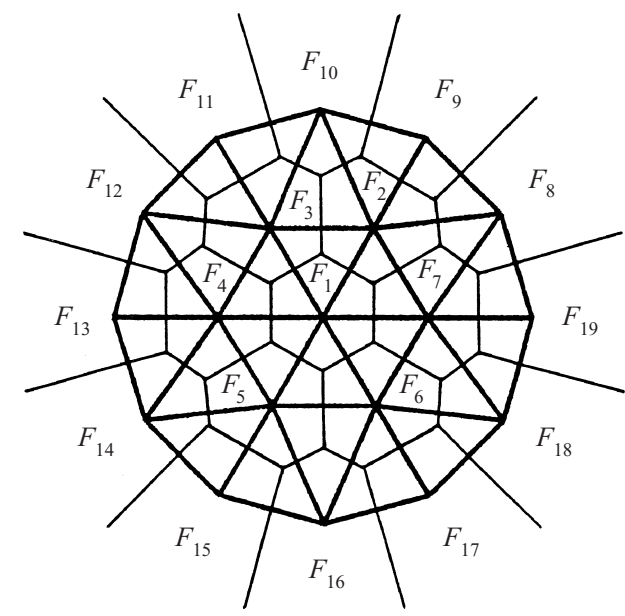

FIGURE 14. The Voronoi tiling and Delaunnay triangulation of $P$ is illustrated for the case $m=6$ and $n=2$. The thin solid lines show the position of the tiles $F_{i}$ and the thick solid lines show the position of the triangles $T_{j}$.

where $d\left(\boldsymbol{x}, \boldsymbol{p}_{k}\right)$ is the distance between position $\boldsymbol{x}$ and the point $\boldsymbol{p}_{k}$. The Voronoi tile $F_{i}$ consists of all the positions $\boldsymbol{x} \in R^{2}$ which are closer to point $\boldsymbol{p}_{i}$ than all the other points $\boldsymbol{p}_{j}$. A globally optimal Delaunnay Triangulation is then constructed by joining all the points $\boldsymbol{p}_{i}$ and $\boldsymbol{p}_{j}$, whose associated tiles $F_{i}$ and $F_{j}$ have a common edge, as shown in figure 14 for the case $m=6, n=2$. The case $m=6$ appears particularly suitable for circular initial geometries, because the triangles naturally form hexagonal groups which tessellate. All the computations presented in this paper have been performed using $m=6$ with ten rings $(n=10)$.

Each triangle is given an index number belonging to the set $M=\{1, \ldots, J\}$, where $J$ equals the total number of triangles in $P$. The set $T=\left\{\boldsymbol{T}_{j}=\left(\alpha_{j}, \beta_{j}, \gamma_{j}\right) j \in M\right\}$ consists of $J$ integer triplets $\left(\alpha_{j}, \beta_{j}, \gamma_{j}\right)$, which exactly define the triangulation of $P$. To construct the finite differences, three sets of geometrical information are required about the relative orientation of the points and the triangular elements:

$$
\begin{aligned}
& N_{i}^{p}=\left\{\boldsymbol{p}_{\alpha_{i}}, \boldsymbol{p}_{\beta_{i}}, \boldsymbol{p}_{\gamma_{i}}, \boldsymbol{p}_{\delta_{i}}, \boldsymbol{p}_{\epsilon_{i}}, \boldsymbol{p}_{\zeta_{i}}\right\} \text { neighbouring points to point } \boldsymbol{p}_{i}, i \in L ; \\
& C_{j}=\left\{\boldsymbol{p}_{\alpha_{j}}, \boldsymbol{p}_{\beta_{3}}, \boldsymbol{p}_{\gamma_{j}}\right\} \text { the corners of each triangle } T_{j}, j \in M ; \\
& N_{i}^{e}=\left\{\boldsymbol{T}_{\alpha_{i}}, \boldsymbol{T}_{\beta_{i}}, \boldsymbol{T}_{\gamma_{i}}, \boldsymbol{T}_{\delta_{i}}, \boldsymbol{T}_{\epsilon_{i}}, \boldsymbol{T}_{\zeta_{i}}\right\} \text { triangles surrounding each } \boldsymbol{p}_{i}, i \in L .
\end{aligned}
$$

The total number of triangles $J=2 I-E-2$ and edges $K=3 I-E-3$ in the triangulation is uniquely determined by the total number of points $I$ in $P$ and the number of edges $E$ of the convex envelope. Each of these sets remains unchanged throughout the numerical simulation.

\section{Appendix C. Volume conservation}

Provided that the thickness $h$ is a smooth scalar field the continuity equation (2.1) can be integrated over any fixed material region (or part) of the avalanche body $\Omega$ by Reynolds' Transport Theorem (e.g. Gurtin 1981), to give

$$
\int_{\Omega}\left\{\frac{\mathrm{d} h}{\mathrm{~d} t}+h\left(\frac{\partial u}{\partial x}+\frac{\partial v}{\partial y}\right)\right\} \mathrm{d} \boldsymbol{x}=\frac{\mathrm{d}}{\mathrm{d} t} \int_{\Omega} h \mathrm{~d} \boldsymbol{x} .
$$


The total volume of granular material $V_{\Omega}$ in $\Omega$ is by definition

$$
V_{\Omega}=\int_{\Omega} h \mathrm{~d} \boldsymbol{x},
$$

and it follows from (2.1) and (C1) that the total volume in any material region of the avalanche body is conserved for all time

$$
\frac{\mathrm{d} V_{\Omega}}{\mathrm{d} t}=0, \quad \Rightarrow \quad V_{\Omega}(t)=V_{\Omega}(0) .
$$

That is the volume $V_{\Omega}(t)$ in any fixed material region $\Omega$ at time $t$ is equal to the initial volume $V_{\Omega}(0)$. In particular (C 3 ) holds for each triangular element $\boldsymbol{T}_{j}, j \in M$, and states that the initial volume contained in the element remains constant for all time.

\section{Appendix D. Earth pressure coefficients}

The Earth pressure coefficients $K_{x}$ and $K_{y}$ are said to be active or passive depending on whether the motion is dilatational or compressional. The following scheme has been used to compute the state of the Earth pressure coefficients. For each triangle $\boldsymbol{T}_{j}\left(\in N_{i}^{e}\right)$ that neighbours point $i$, the positions of the local maximum and minimum $x$ - and $y$-coordinates of the triangles corners are computed:

$$
\left.\begin{array}{ll}
x_{\min _{j}}^{k}=\min \left(x_{\alpha_{j}}^{k}, x_{\beta_{j}}^{k}, x_{\gamma_{j}}^{k}\right), & y_{\min _{j}}^{k}=\min \left(y_{\alpha_{j}}^{k}, y_{\beta_{j}}^{k}, y_{\gamma_{j}}^{k}\right), \\
x_{\text {max }_{j}}^{k}=\max \left(x_{\alpha_{j}}^{k}, x_{\beta_{j}}^{k}, x_{\gamma_{j}}^{k}\right), & y_{\text {max }_{j}}^{k}=\max \left(y_{\alpha_{j}}^{k}, y_{\beta_{j}}^{k}, y_{\gamma_{j}}^{k}\right) .
\end{array}\right\}
$$

These values are used to index the local velocity components at the corners of the triangle $\boldsymbol{T}_{j}$ and hence to compute whether the motion is dilatational or compressional. Equations (2.5) and (2.6) are discretized as follows:

$$
\begin{aligned}
& \left(K_{x}\right)_{j}^{k}= \begin{cases}K_{x_{a c t}} & u\left(x_{\text {max }_{\mathrm{j}}}^{k}\right)-u\left(x_{\text {min }_{\mathrm{j}}}^{k}\right) \geqslant 0 \\
K_{x_{\text {pas }}} & u\left(x_{\text {max }_{\mathrm{j}}}^{k}\right)-u\left(x_{\text {min }_{\mathrm{j}}}^{k}\right)<0,\end{cases}
\end{aligned}
$$

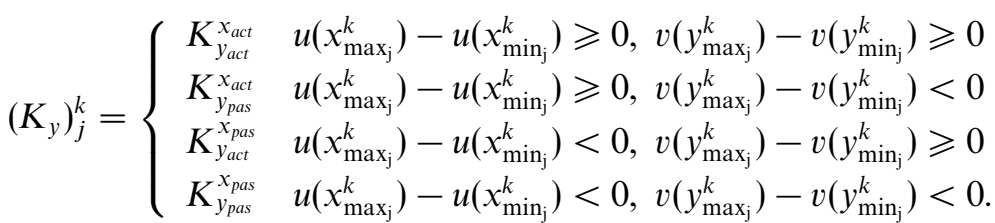

The values of the Earth pressure coefficients $\left(K_{x}\right)_{j}^{k},\left(K_{y}\right)_{j}^{k}$ at the neighbouring triangles $\boldsymbol{T}_{j} \in N_{i}^{e}$ are computed by the algebraic relations (2.7), (2.8). A volume-averaging procedure over the neighbouring triangles is then used to approximate the Earth pressure coefficients at a point

$$
\left(K_{x}\right)_{i}^{k}=\frac{\sum_{j \in N_{i}^{e}}\left(K_{x}\right)_{j}^{k} V_{j}^{0}}{\sum_{j \in N_{i}^{e}} V_{j}^{0}}, \quad\left(K_{y}\right)_{i}^{k}=\frac{\sum_{j \in N_{i}^{e}}\left(K_{y}\right)_{j}^{k} V_{j}^{0}}{\sum_{j \in N_{i}^{e}} V_{j}^{0}} .
$$

This gives larger elements more weight in determining the value of the Earth pressure coefficient.

\section{Appendix E. Free-surface gradients}

The free-surface gradients $(\partial s / \partial x)_{i}^{k},(\partial s / \partial y)_{i}^{k}$ at each point $i \in L$ must be computed in order to calculate the thickness gradients in (4.6) and (4.7). A volume-averaging 


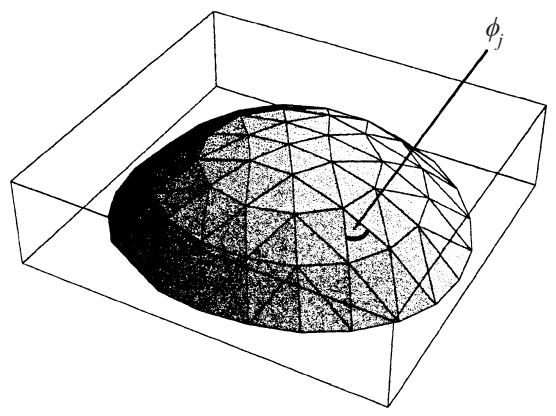

FIGURE 15. The avalanche free surface is approximated by triangular plane elements between each grid cell. The angle $\phi_{j}$ is used as a weighting factor to calculate the average free surface gradient at a grid point.

procedure similar to that used in computing the average Earth pressure coefficients is used to construct the thickness $h_{i}^{k}$ at a point from the thicknesses $h_{j}^{k}$ at the neighbouring elements $j \in N_{i}^{e}$ :

$$
h_{i}^{k}=\frac{\sum_{j \in N_{i}^{e}} h_{j}^{k} V_{j}^{0}}{\sum_{j \in N_{i}^{e}} V_{j}^{0}} .
$$

It follows for the free surface at a point that

$$
s_{i}^{k}=h_{i}^{k}+b\left(x_{i}^{k}, y_{i}^{k}\right) .
$$

The free-surface gradients $(\partial s / \partial x)_{j}^{k},(\partial s / \partial y)_{j}^{k}$ at an element $j$ are then constructed by calculating the slope of a plane passing through its corners $\boldsymbol{p}_{\alpha_{j}}, \boldsymbol{p}_{\beta_{j}}, \boldsymbol{p}_{\gamma_{j}}$. This implies

$$
\begin{aligned}
& \left(\frac{\partial s}{\partial x}\right)_{j}^{k}=\frac{s_{\alpha_{j}}\left(y_{\beta_{j}}^{k}-y_{\gamma_{j}}^{k}\right)+s_{\beta_{j}}\left(y_{\gamma_{j}}^{k}-y_{\alpha_{j}}^{k}\right)+s_{\gamma_{j}}\left(y_{\alpha_{j}}^{k}-y_{\beta_{j}}^{k}\right)}{x_{\alpha_{j}}\left(y_{\beta_{j}}^{k}-y_{\gamma_{j}}^{k}\right)+x_{\beta_{j}}\left(y_{\gamma_{j}}^{k}-y_{\alpha_{j}}^{k}\right)+x_{\gamma_{j}}\left(y_{\alpha_{j}}^{k}-y_{\beta_{j}}^{k}\right)}, \\
& \left(\frac{\partial s}{\partial y}\right)_{j}^{k}=\frac{s_{\alpha_{j}}\left(x_{\beta_{j}}^{k}-x_{\gamma_{j}}^{k}\right)+s_{\beta_{j}}\left(x_{\gamma_{j}}^{k}-x_{\alpha_{j}}^{k}\right)+s_{\gamma_{j}}\left(x_{\alpha_{j}}^{k}-x_{\beta_{j}}^{k}\right)}{y_{\alpha_{j}}\left(x_{\beta_{j}}^{k}-x_{\gamma_{j}}^{k}\right)+y_{\beta_{j}}\left(x_{\gamma_{j}}^{k}-x_{\alpha_{j}}^{k}\right)+y_{\gamma_{j}}\left(x_{\alpha_{j}}^{k}-x_{\beta_{j}}^{k}\right)} .
\end{aligned}
$$

These free-surface gradients then have to be averaged again to obtain $(\partial s / \partial x)_{i}^{k}$, $(\partial s / \partial y)_{i}^{k}$ defined at each point $i \in L$. That is

$$
(\partial s / \partial x)_{i}^{k}=\frac{\sum_{j \in N_{i}^{e}}(\partial s / \partial x)_{j}^{k} W_{j}}{\sum_{j \in N_{i}^{e}} W_{j}}, \quad(\partial s / \partial y)_{i}^{k}=\frac{\sum_{j \in N_{i}^{e}}(\partial s / \partial y)_{j}^{k} W_{j}}{\sum_{j \in N_{i}^{e}} W_{j}},
$$

where $W_{j}=\left|\phi_{j}\right|$ is an angle weighting factor. The angle $\phi_{j}$ is the angle that the free-surface triangle, $j$, makes at the point $\boldsymbol{p}_{i}$. This is shown in figure 15. Assuming the other vertices of the triangle are $\boldsymbol{p}_{\phi}, \boldsymbol{p}_{\chi} \in C_{j}$ the weighting factor is

$$
W_{j}=\left|\cos ^{-1}(\boldsymbol{a} \cdot \boldsymbol{b} /(|\boldsymbol{a}||\boldsymbol{b}|))\right|,
$$

where $\boldsymbol{a}=\left(x_{\phi}-x_{i}, y_{\phi}-y_{i}, s_{\phi}-s_{i}\right)$ and $\boldsymbol{b}=\left(x_{\chi}-x_{i}, y_{\chi}-y_{i}, s_{\chi}-s_{i}\right)$. This method enhances the stability of the numerical algorithm.

\section{Appendix F. Artificial viscosity}

Although the Savage-Hutter equations (2.1)-(2.3) appear relatively simple they have proved particularly difficult to integrate numerically. Even in the one-dimensional 


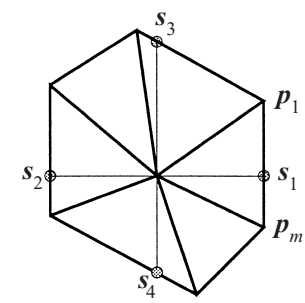

FIGURE 16. Construction of the intersection points in the interior of the avalanche domain.

Lagrangian method developed by Savage \& Hutter (1989), some additional artificial numerical diffusion was necessary. This is because shocks can occur when there is rapid convergence of the granular material, which the numerical method cannot handle. Artificial viscosity terms $\Psi_{x}=\mu_{x} \partial^{2} u / \partial x^{2}$ and $\Psi_{y}=\mu_{y} \partial^{2} v / \partial y^{2}$ are therefore added to the right-hand sides of the momentum balance equations (2.2) and (2.3) to diffuse the sharp gradients in thickness and velocity that develop. Two different viscosities $\mu_{x}$ and $\mu_{y}$ are used in the $x$ - and $y$-directions. For the simulations presented in this paper the diffusion terms are only activated after the tenth non-dimensional time unit as they are not needed to maintain stability when the avalanche is diverging.

To compute the $x$-derivatives at interior points two intersection points $\boldsymbol{s}_{1}=$ $\left(x\left(s_{1}\right)_{i}^{k}, y_{i}^{k}\right)$ and $\boldsymbol{s}_{2}=\left(x\left(s_{2}\right)_{i}^{k}, y_{i}^{k}\right)$ are first constructed. These both lie on the line $y=y_{i}^{k}$ but on opposite sides of $x=x_{i}^{k}$. The components $x\left(s_{1}\right)$ and $x\left(\boldsymbol{s}_{2}\right)$ are defined by the intersection of $y=y_{i}^{k}$ with the convex edge formed by the set of points $N_{i}^{p}$. More precisely, for the intersection point $s_{1}$ a search is performed to determine the points $\boldsymbol{p}_{l}, \boldsymbol{p}_{m} \in N_{i}^{p}$ that lie immediately above and below the line $y=y_{i}^{k}$. A straight line is then drawn between the two points and the $x$-component at the intersection with the line $y=y_{i}^{k}$ is calculated by

$$
x\left(s_{1}\right)=\frac{x_{l}-x_{m}}{y_{l}-y_{m}}\left(y_{i}-y_{l}\right)+x_{l} .
$$

This is illustrated in figure 16. A linear interpolation is then used to approximate the downslope and cross-slope velocity components at the intersection point:

$$
u\left(\boldsymbol{s}_{1}\right)=\frac{\left|\boldsymbol{s}_{1}-\boldsymbol{p}_{l}\right|}{\left|\boldsymbol{p}_{l}-\boldsymbol{p}_{m}\right|}\left(u_{l}-u_{m}\right)+u_{l}, \quad v\left(\boldsymbol{s}_{1}\right)=\frac{\left|\boldsymbol{s}_{1}-\boldsymbol{p}_{l}\right|}{\left|\boldsymbol{p}_{l}-\boldsymbol{p}_{m}\right|}\left(v_{l}-v_{m}\right)+v_{l},
$$

where $\left(u_{l}, v_{l}\right)$ and $\left(u_{m}, v_{m}\right)$ are the velocities at $\boldsymbol{p}_{l}$ and $\boldsymbol{p}_{m}$, respectively. An analogous method is used to find the other intersection point $x\left(\boldsymbol{s}_{2}\right) \neq x\left(\boldsymbol{s}_{1}\right)$ and the velocity $\boldsymbol{v}\left(\boldsymbol{s}_{2}\right)$. In the $y$-direction two new intersection points $s_{3}, s_{4}$ and their velocities are defined in a similar manner except that this time they lie on the line $x=x_{i}^{k}$.

At the boundary of the avalanche the edge points are not surrounded by a complete six-element net. Instead there may be either three neighbouring grid points (and two elements) or four neighbouring points (and three elements) depending on position. The construction of the intersection points at the edge is not possible, as at least one of these points will lie outside the avalanche domain. For this reason virtual grid points are defined outside the avalanche domain as shown in figure 17. These virtual grid points have no dynamics of their own and act merely as a means of constructing the intersection points and thereby the finite difference approximations at the edge of the domain. The virtual grid points for edge point $\boldsymbol{p}_{i}$ are constructed by interpolating 
(a)

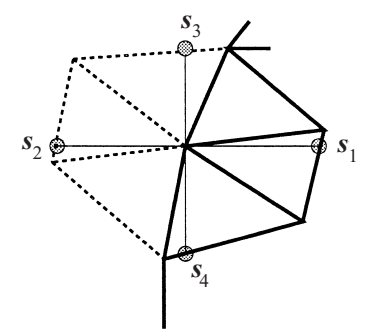

(b)

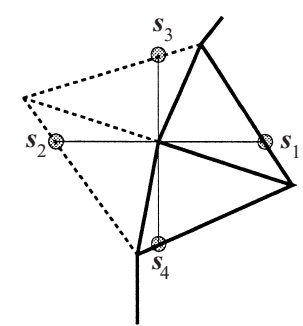

FIGURE 17. Construction of the intersection points at the edge of the avalanche with $(a)$ three neighbouring elements and $(b)$ two neighbouring elements. The dashed lines indicate the position of the virtual grid edges.

the position of an internal neighbouring point $\boldsymbol{p}_{n}$ to a position $\hat{\boldsymbol{p}}_{n}$ outside the domain

$$
\left(\hat{x}_{n}\right)_{i}^{k}=2 x_{i}^{k}-x_{n}^{k}, \quad\left(\hat{y}_{n}\right)_{i}^{k}=2 y_{i}^{k}-y_{n}^{k} .
$$

When there are three neighbouring grid points only one of these is internal and therefore only one virtual grid point is constructed, and when there are four neighbouring points two of these are internal and therefore two virtual points are constructed. This is shown in figure 17. The velocity of the virtual points is then computed by a Taylor expansion about point $\boldsymbol{p}_{n}$. The intersection points at the edge and their velocities can then be constructed in the same manner as for the internal points.

Having constructed the position and velocity of the intersection points for all points within the avalanche grid net, the finite difference approximations to the diffusion terms are

$$
\left.\begin{array}{l}
\left(\Psi_{x}\right)_{i}^{k}=\mu_{x}\left(\left(a_{x}\right)_{i}^{k} u\left(s_{1}\right)_{i}^{k-1 / 2}+\left(b_{x}\right)_{i}^{k} u_{i}^{k-1 / 2}+\left(c_{x}\right)_{i}^{k} u\left(s_{2}\right)_{i}^{k-1 / 2}\right), \\
\left(\Psi_{y}\right)_{i}^{k}=\mu_{y}\left(\left(a_{y}\right)_{i}^{k} v\left(\boldsymbol{s}_{3}\right)_{i}^{k-1 / 2}+\left(b_{y}\right)_{i}^{k} v_{i}^{k-1 / 2}+\left(c_{y}\right)_{i}^{k} v\left(\boldsymbol{s}_{4}\right)_{i}^{k-1 / 2}\right),
\end{array}\right\}
$$

with coefficients

$$
\begin{aligned}
& \left(a_{x}\right)_{i}^{k}=\frac{-2}{\left(x_{i}^{k}-x\left(s_{1}\right)_{i}^{k}\right)\left(x\left(s_{1}\right)_{i}^{k}-x\left(s_{2}\right)_{i}^{k}\right)}, \quad\left(a_{y}\right)_{i}^{k}=\frac{-2}{\left(y_{i}^{k}-y\left(s_{3}\right)_{i}^{k}\right)\left(y\left(s_{3}\right)_{i}^{k}-y\left(s_{4}\right)_{i}^{k}\right)}, \\
& \left(b_{x}\right)_{i}^{k}=\frac{2}{\left(x_{i}^{k}-x\left(s_{1}\right)_{i}^{k}\right)\left(x_{i}^{k}-x\left(s_{2}\right)_{i}^{k}\right)}, \quad\left(b_{y}\right)_{i}^{k}=\frac{2}{\left(y_{i}^{k}-y\left(s_{3}\right)_{i}^{k}\right)\left(y_{i}^{k}-y\left(s_{4}\right)_{i}^{k}\right)}, \\
& \left(c_{x}\right)_{i}^{k}=\frac{2}{\left(x_{i}^{k}-x\left(s_{2}\right)_{i}^{k}\right)\left(x\left(s_{1}\right)_{i}^{k}-x\left(s_{2}\right)_{i}^{k}\right)}, \quad\left(c_{y}\right)_{i}^{k}=\frac{2}{\left(y_{i}^{k}-y\left(s_{4}\right)_{i}^{k}\right)\left(y\left(s_{3}\right)_{i}^{k}-y\left(s_{4}\right)_{i}^{k}\right)} \text {. }
\end{aligned}
$$

This completes the finite difference approximation of (4.6) and (4.7).

\section{REFERENCES}

Buchholz, A. \& RÜGER, W. 1979 Photogrammetrie. VEB Velag für Bauwesen, Berlin.

Gray, J. M. N. T. \& Hutter, K. 1997 Pattern formation in granular avalanches. Contin. Mech. Thermodyn. 9, 341-345.

Gray, J. M. N. T. \& Hutter, K. 1998 Physik granularer Lawinen. Physik. Blätter 54, 37-43.

Gray, J. M. N. T., Wieland, M. \& Hutter, K. 1999 Gravity driven free surface flow of granular avalanches over complex basal topography. Proc. R. Soc. Lond. A 455, 1841.

Greve, R. \& Hutter, K. 1993 Motion of a granular avalanche in a convex and concave curved chute: experiments and theoretical predictions. Phil. Trans. R. Soc. Lond. A 342, 573-600. 
Greve, R., Koch, T. \& Hutter, K. 1994 Unconfined flow of granular avalanches along a partly curved surface. Part I: Theory. Proc. R. Soc. Lond. A 445, 399-413.

Gurtin, M. E. 1981 An Introduction to Continuum Mechanics. Academic.

Hoschek, J. \& Lasser, D. 1992 Grundlagen der Geometrischen Datenverarbeitung. B. G. Teubner, Stuttgart.

Hungr, O. \& Morgenstern, N. R. 1984a Experiments on the flow behaviour of granular materials at high velocity in an open channel flow. Geotechnique 34, 405-413.

Hungr, O. \& Morgenstern, N. R. 1984b High velocity ring shear tests on sand. Geotechnique 34, 415-421.

Hutter, K. \& Koch, T. 1991 Motion of a granular avalanche in an exponentially curved chute: experiments and theoretical predictions. Phil. Trans. R. Soc. Lond. A 334, 93-138.

Hutter, K., Siegel, M., Savage, S. B. \& Nohguchi, Y. 1993 Two-dimensional spreading of a granular avalanche down an inclined plane. Part I: Theory. Acta Mech. 100, 37-68.

Jenkins, J. T. \& Savage, S. B. 1983 A theory for the rapid flow of identical, smooth, nearly elastic spherical particles. J. Fluid Mech. 130, 187-202.

Koch, T., Greve, R. \& Hutter, K. 1994 Unconfined flow of granular avalanches along a partly curved surface, Part II: Experiments and numerical computations. Proc. R. Soc. Lond. A 445, 415-435.

Kraus, K. 1982 Band 1. Grundlagen und Standardverfahren. F. Dümmlers Verlag, Bonn.

REYNOLDS, O. 1885 On the dilatancy of media composed of rigid particles in contact. Phil. Mag. (5) 20, 469-481.

Savage, S. B. \& Hutter, K. 1989 The motion of a finite mass of granular material down a rough incline. J. Fluid Mech. 199, 177-215.

Savage, S. B. \& Hutter, K. 1991 The dynamics of avalanches of granular materials from initiation to runout. Part I: Analysis. Acta Mech. 86, 201-223. 